MATHEMATICS OF COMPUTATION

Volume 75, Number 255, July 2006, Pages 1319-1349

S 0025-5718(06)01828-X

Article electronically published on May 3, 2006

\title{
WAVELETS ON MANIFOLDS: AN OPTIMIZED CONSTRUCTION
}

\author{
ANGELA KUNOTH AND JAN SAHNER
}

Dedicated to Peter Deuflhard on the occasion of his 60th birthday

\begin{abstract}
A key ingredient of the construction of biorthogonal wavelet bases for Sobolev spaces on manifolds, which is based on topological isomorphisms is the Hestenes extension operator. Here we firstly investigate whether this particular extension operator can be replaced by another extension operator. Our main theoretical result states that an important class of extension operators based on interpolating boundary values cannot be used in the construction setting required by Dahmen and Schneider. In the second part of this paper, we investigate and optimize the Hestenes extension operator. The results of the optimization process allow us to implement the construction of biorthogonal wavelets from Dahmen and Schneider. As an example, we illustrate a wavelet basis on the 2 -sphere.
\end{abstract}

\section{INTRODUCTION}

This paper is motivated by the results presented in [DS]. There compactly supported biorthogonal wavelet bases for Sobolev spaces $H^{m}(\Gamma)$ on manifolds $\Gamma$ were constructed which may be used, for instance, to numerically solve boundary integral equations by wavelet schemes; see, e.g., $\mathrm{Ha}$. The starting point is a quadrangulation for $\Gamma$, i.e., $\Gamma=\bigcup_{i=1}^{N} \overline{\Gamma_{i}}$ for $\Gamma_{i}:=\kappa_{i}(\square)$ being the parametric image of $\square=(0,1)^{d}$ under $\kappa_{i}$, and $\Gamma_{i} \cap \Gamma_{j}=\emptyset$ for $i \neq j$. The construction in DS] is based on a topological isomorphism

$$
P: \prod_{i=1}^{N} H^{m}\left(\Gamma_{i}\right)^{\uparrow} \rightarrow H^{m}(\Gamma)
$$

developed previously in $\mathrm{CF}$ that yields a decomposition of the Sobolev space $H^{m}(\Gamma)$ into particular closed subspaces $H^{m}\left(\Gamma_{i}\right)^{\uparrow}$ of $H^{m}(\Gamma)$. Here any $m$ is allowed as long as it does not exceed the smoothness of the manifold. Using this decomposition, norm equivalences for $H^{m}(\Gamma)$ in terms of weighted sequence norms of coefficients of wavelet expansions were given in DS for the whole range of admissible $m$. Such norm equivalences are fundamental for applications of wavelet bases. Other constructions based on domain decomposition and gluing [CTU, DS1] only

Received by the editor July 30, 2004 and, in revised form, April 16, 2005.

2000 Mathematics Subject Classification. Primary 65T60, 54C20; Secondary 42C40, 34B05.

Key words and phrases. Wavelets on manifolds, topological isomorphisms, extension operators, optimized Hestenes extension, trace dependent operators, biorthogonal wavelets, 2-sphere.

This work has been supported by the Deutsche Forschungsgemeinschaft (SFB 611) at the Universität Bonn.

(C)2006 American Mathematical Society Reverts to public domain 28 years from publication 

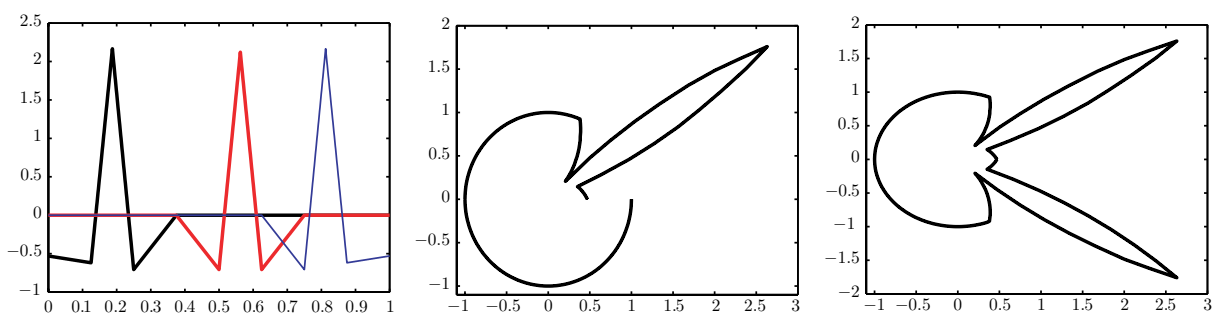

FiguRE 1. Left: Boundary adapted and interior wavelets on the interval from [DKU]. Middle: Right boundary adapted wavelet from the left figure pushed forward to the upper part of the circle $S^{1}$ and extended by zero onto the lower part. Right: Same wavelet pushed extended continuously.

achieve norm equivalences $-\frac{1}{2}<m<\frac{3}{2}$ for arbitrary manifolds, or $|m| \leq 1$ for the special case of piecewise affine surfaces with triangular facets DSt].

Due to the structure of (1.1), wavelet bases $\Psi$ of $H^{m}(\square)$ will serve as the first basic ingredient. Such bases were constructed in DKU] (see the left plot in Figure 11). These wavelets are pushed forward to the patches $\Gamma_{i}$ first to obtain wavelet bases $\Psi_{\Gamma_{i}}$ of $H^{m}\left(\Gamma_{i}\right)^{\uparrow}$. Defining $\Psi_{\Gamma_{i}}^{\prime}:=\Psi \circ \kappa_{i}^{-1}$ and extending $\Psi_{\Gamma_{i}}^{\prime}$ by zero outside of $\Gamma_{i}$, we see, however, that the collection

$$
\Psi^{\prime}:=\Psi_{\Gamma_{1}}^{\prime} \cup \cdots \cup \Psi_{\Gamma_{N}}^{\prime}
$$

is not a candidate for a basis of $H^{m}(\Gamma)$ for $m>\frac{1}{2}$ : for some $\psi \in \Psi$ not vanishing on parts of the boundary $\partial \square$ of $\square, \psi \circ \kappa_{i}^{-1}$ is not continuous in $\Gamma$ and, hence, $\psi \circ \kappa_{i}^{-1} \notin H^{m}(\Gamma)$. In the middle figure in Figure 1 the right boundary wavelet from the left plot is pushed forward to the circle $\Gamma:=S^{1}$ and quadrangulated into the upper and lower half of the circle. It is obviously not continuous in $S^{1}$. Extending it continuously onto the lower part of $S^{1}$ yields the figure on the right of Figure 1 , Hence, the main task of the operator $P$ in (1.1) is to extend the single components $\Psi_{i} \in H^{m}\left(\Gamma_{i}\right)^{\uparrow}$ onto the whole of $\Gamma$ in some appropriate way. This is done by certain extension operators which play the role of the second main ingredient for constructing wavelet bases on manifolds. Recall that for some open subset $\Omega \subset \Gamma$, $E: H^{m}(\Omega) \rightarrow H^{m}(\Gamma)$ is called an extension operator if $\left.E f\right|_{\Omega}=f$ is satisfied for all $f \in H^{m}(\Omega)$, where $\left.\right|_{\Omega}$ denotes the restriction to $\Omega$. Given such extension operators

$$
E_{i}: H^{m}\left(\Gamma_{i}\right) \rightarrow H^{m}(\Gamma),
$$

the collection $\Psi_{\Gamma}:=\Psi_{\Gamma_{1}} \cup \cdots \cup \Psi_{\Gamma_{N}}$ with $\Psi_{\Gamma_{i}}:=E_{i}\left(\Psi \circ \kappa_{i}^{-1}\right)$ is a reasonable candidate for a basis of $H^{m}(\Gamma)$. For the example discussed before, the right figure in Figure 1 displays a possible extension to the whole of $S^{1}$.

To establish the equivalence of norms for the isomorphism (1.1), the extension operators $E_{i}$ as well as their adjoints $E_{i}^{*}$ relative to the $L^{2}$ inner product have to satisfy quite severe conditions, denoted as property (E):

(CE) Continuity: $E_{i}: H^{m}\left(\Gamma_{i}\right) \rightarrow H^{m}(\Gamma)$ is continuous.

$\left(\mathrm{CE}^{*}\right)$ Continuity of the $L^{2}$ adjoint relative to $H^{m}$ : The $L^{2}$ adjoint $E_{i}^{*}: L^{2}(\Gamma) \rightarrow$ $L^{2}\left(\Gamma_{i}\right)$ of $E_{i}$ is well defined and continuous as an operator $E_{i}^{*}: H^{m}(\Gamma) \rightarrow$ $H^{m}\left(\Gamma_{i}\right)$; i.e., $\left\|E_{i}^{*} f\right\|_{H^{m}\left(\Gamma_{i}\right)} \lesssim\|f\|_{H^{m}(\Gamma)}$ for all $f \in H^{m}(\Gamma)$. 
Here and in the rest of the paper $a \lesssim b$ means $a \leq c b$ with some constant $c$ which is independent of all parameters on which $a$ and $b$ may depend. An operator that meets these requirements is the Hestenes extension operator $[\mathrm{H}]$ used in [DS]. However, a brief look reveals that this operator is initially ill conditioned-it produces strongly oscillating extensions; see Section 6 . This fact leads to large constants in the norm equivalence of the isomorphism (1.1).

The main objective of this paper is the construction of well-conditioned extension operators satisfying (E), which makes the implementation of wavelets on manifolds practically feasible. Firstly, this motivates the search for alternative extension operators in Sections 3 and 4 . We propose two different such operators, both by interpolating trace values at the boundary: the first of such trace dependent operators is based on Taylor expansions, the second employs norm minimizing harmonic extensions, and both satisfy (CE). However, as stated in the main theoretical result of this paper, Theorem 4.7, no extension operator based on the interpolation of boundary values can be further employed as their adjoints do not satisfy $\left(\mathrm{CE}^{*}\right)$. Nevertheless, these results lead us to finding an extension operator that does not depend on the values of the trace directly. A variant of the Hestenes operator employed in DS turns out to be our method of choice which is derived in Section 5 where we also prove that the Hestenes extension emerges as a limit of an interpolation process with polynomials. In particular, we realize $H^{m}$, instead of $C^{m}$, extensions. In Section 6 the Hestenes extension is examined thoroughly: we perform a number of modifications which substantially reduce the oscillations of the extension and optimize its ingredients in Section 7. The construction of wavelets from [DS] is summarized in Section 8, An implementation of the construction employing the optimized Hestenes extension is based on the software package IGPMLIB IGPM] t the resulting $\mathrm{C}++$ library is documented in $[\mathrm{S}$. This library is finally used in Section 9 to construct and visualize nontrivial application biorthogonal wavelet bases for $H^{1}\left(S^{2}\right)$ on the sphere $S^{2} \subset \mathbb{R}^{3}$ based on an appropriate quadrangulation of $S^{2}$.

\section{Some BASIC NOTIONS}

In the rest of this paper, we will only consider extensions of functions in Sobolev spaces of natural order $m \in \mathbb{N}$; see, e.g., A for a general reference. For an open interval $I \subset \mathbb{R}$ and $m \in \mathbb{N}, 1 \leq p \leq \infty, f \in L^{p}(I)$ has weak derivatives of order $m$ in $L^{p}(I)$ if for all $k \in \mathbb{N}$ with $k \leq m$ there exist functions $f^{(k)} \in L^{p}(I)$ satisfying

$$
\int_{I} f(x) \partial_{x}^{k} \varphi(x) d x=(-1)^{k} \int_{I} f^{(k)}(x) \varphi(x) d x
$$

for all $\varphi \in C_{0}^{\infty}(I)$. Here $C_{0}^{\infty}(I)$ denotes the space of arbitrarily smooth functions with compact support in $I$. The spaces

$$
W^{m, p}(I):=\left\{f \in L^{p}(I): f \text { has weak derivatives } f^{(k)} \in L^{p}(I) \text { of order } m\right\}
$$

are the Sobolev spaces of order $m$, equipped with the norm

$$
\|f\|_{W^{m, p}(I)}:=\sum_{k=0}^{m}\left\|f^{(k)}\right\|_{L^{p}(I)}
$$


and the norm in $H^{m}(I):=W^{m, 2}(I)$ is induced by the inner product

$$
\langle f, g\rangle_{H^{m}(I)}:=\sum_{k=0}^{m}\left\langle f^{(k)}, g^{(k)}\right\rangle_{L^{2}(I)} .
$$

Moreover, zero boundary values are incorporated into the vector space $H_{0}^{m}(I)$ by defining $H_{0}^{m}(I)$ as the closure of $C_{0}^{\infty}(I)$ with respect to the norm in $H^{m}(I)$. In order to be able to assign boundary values to $f \in H^{m}(I)$ we recall

Theorem 2.1. For $m \geq 1$ the embedding operator $H^{m}(I) \hookrightarrow C^{m-1}(\bar{I})$ is well defined and continuous. In particular, $f \in H^{m}(I)$ can be assigned boundary values $\left.f^{(k)}\right|_{\partial I}$ for all $k=0, \ldots, m-1$, called the trace values of $f$.

As we will see below, the extension operators will be defined by gluing together functions defined on adjacent intervals.

Remark 2.2. Let $I_{1}, I_{2} \subset \mathbb{R}$ be open intervals satisfying $\overline{I_{1}} \cap \overline{I_{2}}=\{b\}$. Then one has $f_{1} \in H^{m}\left(I_{1}\right), f_{2} \in H^{m}\left(I_{2}\right), f_{1}^{(k)}(b)=f_{2}^{(k)}(b)$ if and only if $\chi_{I_{1}} f_{1}+\chi_{I_{2}} f_{2} \in$ $H^{m}\left(I_{1} \cup\{b\} \cup I_{2}\right)$. Here for any interval $I, \chi_{I}$ denotes the characteristic function on $I$. By $\chi_{I}$ we sometimes also denote the extension by zero to some set $J \supset I$ which will be clear from the context. Recall also that for $I \subset \mathbb{R}$ an open interval, one has $f \in H_{0}^{m}(I)$ if and only if $f \in H^{m}(I)$ and $\left.f^{(k)}\right|_{\partial I}=0$ for all $k=0, \ldots, m-1$.

\section{UNIVARIATE EXTENSION OPERATORS}

The multivariate extension operators needed later in Subsection 8.5 can be defined as tensor products of univariate extension operators $A$. We use the following notation. For $a<b<c<d$, let intervals $I, \tilde{I} \subset \mathbb{R}$ be defined by $I:=(b, c), \tilde{I}:=(a, d)$. The boundaries of $I, \tilde{I}$ will be denoted by $\partial I=\{b, c\}$, $\partial \tilde{I}=\{a, d\}$, respectively. An operator $A: H^{m}(I) \rightarrow H^{m}(\tilde{I})$ is called a univariate extension operator if $A$ satisfies $\left.(A f)\right|_{I}=f$ for all $f \in H^{m}(I)$. We first boil down the formulation of the crucial property (E) to the univariate case. We say that a univariate extension operator $A$ satisfies property (A) if

(L) Locality: For $f \in H^{m}(I)$ one has $A f \in H_{0}^{m}(\tilde{I})$.

(CA) Continuity: $A: H^{m}(I) \rightarrow H_{0}^{m}(\tilde{I})$ is continuous.

$\left(\mathrm{CA}^{*}\right)$ Continuity of $A^{*}: H^{m}(\tilde{I}) \rightarrow H^{m}(I)^{*}$, defined by $\left\langle A^{*} g, f\right\rangle:=\langle g, A f\rangle_{L^{2}(\tilde{I})}$ for all $f \in H^{m}(I), g \in H^{m}(\tilde{I})$, is continuous as an operator $A^{*}: H^{m}(\tilde{I}) \rightarrow$ $H^{m}(I)$ with the adjoint property

$$
\left\langle A^{*} g, f\right\rangle=\left\langle A^{*} g, f\right\rangle_{L^{2}(I)}=\langle g, A f\rangle_{L^{2}(\tilde{I})} .
$$

Of course, $\left(\mathrm{CA}^{*}\right)$ is a stronger requirement than $A^{*}$ to be continuous as an operator $H^{m}(\tilde{I}) \rightarrow H^{m}(I)^{*}$. Here and below, for a vector space $V$, we denote its dual by $V^{*}$, the space of continuous linear functionals $V \rightarrow \mathbb{R}$, and $\langle\cdot, \cdot\rangle$ denotes the dual pairing, which is given by $\langle F, f\rangle:=F(f)$ for all $F \in V^{*}, f \in V$.

The detailed discussion of univariate extension operators will be of vital importance for the multivariate case in Subsection 8.5 below and requires the effort of Sections 47 Next we discuss two classes of univariate extension operators. 


\section{EXTENSION OPERATORS BASED ON TRACES}

Remark 2.2 enables us to extend functions by interpolating the first $m-1$ derivatives of $f \in H^{m}(I)$ explicitly at the boundary $\partial I$. As we are interested in extensions $A f \in H_{0}^{m}(\tilde{I})$, we also have to achieve zero boundary values in the $H^{m}$-sense at the boundary $\partial \tilde{I}$. Writing $I_{\ell}:=(a, b), I_{r}:=(c, d)$, we have to find functions $u_{\ell} \in H^{m}\left(I_{\ell}\right), u_{r} \in H^{m}\left(I_{r}\right)$ that satisfy the boundary conditions

$$
\begin{array}{ll}
u_{\ell}{ }^{(k)}(a)=0, u_{\ell}{ }^{(k)}(b)=f^{(k)}(b) & \text { for } k=0, \ldots, m-1, \\
u_{r}{ }^{(k)}(d)=0, u_{r}{ }^{(k)}(c)=f^{(k)}(c) & \text { for } k=0, \ldots, m-1 .
\end{array}
$$

Definition 4.1. Let $A: H^{m}(I) \rightarrow H^{m}(\tilde{I})$ be a continuous extension operator. Then $A$ is called trace dependent if for all $f \in H^{m}(I)$ the definition of $A f$ depends only on the trace values $f^{(k)}(b), f^{(k)}(c), k=0, \ldots, m-1$, of $f$ at the boundary $\partial I$.

Since trace dependent operators by their very definition require the availability of the trace values of the derivatives of $f$ which are not always accessible, they are of limited applicability. However, these extensions are of theoretical interest and some of their properties will be exploited further on. We now discuss two different solutions to this interpolation problem which only employ values of $f$ at $\partial I$. In contrast, we will later consider approaches where, in addition, values of $f$ in the interior of $I$ come into play. Note that, due to Theorem 2.1 $f^{(m-1)} \in H^{1}(I)$ is still continuous, but not necessarily $f^{(m)}$. Thus, interpolation of the first $m$ derivatives can in general not be carried out for $H^{m}$-functions. At first we look at an operator based on a Taylor expansion.

4.1. Extension based on a Taylor expansion. Let $\eta \in C_{0}^{\infty}(\tilde{I})$ be a cutoff function displayed in Figure 2 which is defined by

$$
\eta(x)= \begin{cases}0, & x \in(a, d) \backslash\left[\frac{a+b}{2}, \frac{c+d}{2}\right], \\ 1, & x \in\left[\frac{a+3 b}{4}, \frac{3 c+d}{4}\right] .\end{cases}
$$

Lemma 4.2. The operator

$$
\begin{aligned}
\left(E_{T} f\right)(x):= & \chi_{I_{\ell}}(x) \eta(x) \sum_{k=0}^{m-1} \frac{f^{(k)}(b)}{k !}(x-b)^{k}+\chi_{I}(x) f(x) \\
& +\chi_{I_{r}}(x) \eta(x) \sum_{k=0}^{m-1} \frac{f^{(k)}(c)}{k !}(x-c)^{k}
\end{aligned}
$$

is a continuous linear extension operator $E_{T}: H^{m}(I) \rightarrow H_{0}^{m}(\tilde{I})$.

The proof is straightforward and can be found in [KS.

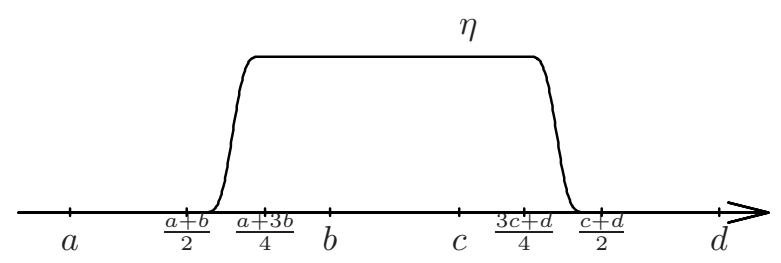

FiguRE 2. The cutoff function $\eta$. 
4.2. A norm minimizing extension. Another possible extension is based on finding functions $u_{\ell} \in H^{m}\left(I_{\ell}\right), u_{r} \in H^{m}\left(I_{r}\right)$ which minimize the functionals

$$
\tilde{u} \mapsto\|\tilde{u}\|_{H^{m}\left(I_{\ell}\right)}, \quad \tilde{u} \mapsto\|\tilde{u}\|_{H^{m}\left(I_{r}\right)}
$$

among all functions $\tilde{u}$ satisfying the boundary conditions (4.1), (4.2), respectively. Clearly, defining

$$
u_{\ell}:=\underset{\tilde{u} \in H^{m}\left(I_{\ell}\right), \tilde{u} \text { satisfies (4.1) }}{\operatorname{argmin}}\|\tilde{u}\|_{H^{m}\left(I_{\ell}\right)}^{2}, \quad u_{r}:=\underset{\tilde{u} \in H^{m}\left(I_{r}\right), \tilde{u} \text { satisfies (4.2) }}{\operatorname{argmin}}\|\tilde{u}\|_{H^{m}\left(I_{r}\right)}^{2},
$$

the operator $E$ defined by

$$
(E f)(x):=\chi_{I_{\ell}}(x) u_{\ell}(x)+\chi_{I}(x) f(x)+\chi_{I_{r}}(x) u_{r}(x), \quad E: H^{m}(I) \rightarrow H^{m}(\tilde{I}),
$$

is the operator with the smallest operator norm among all possible extension operators. We restrict the our discussion to satisfying (4.1). As for (4.2) the situation is analogous, and the following results were established in [KS], where also details of the explicit construction can be found.

Lemma 4.3. The extension operator $E: H^{m}(I) \rightarrow H^{m}(\tilde{I})$ defined in (4.4) satisfies $\|E f\|_{H^{m}(\tilde{I})} \lesssim\|f\|_{H^{m}(I)}$ for all $f \in H^{m}(I)$.

As an application of the extension operator $E$ defined in (4.4) we will later need norm minimizing cutoff functions. By this we mean for $a<b \leq c<d$ a function $\eta \in H^{m}(\mathbb{R})$ satisfying $|\eta(x)| \leq 1$ and

$$
\eta(x)= \begin{cases}0, & x \in \mathbb{R} \backslash \tilde{I}, \\ 1, & x \in I,\end{cases}
$$

with minimal $H^{m}$-norm. Using $E$ we extend the function $f: I \rightarrow \mathbb{R}, f \equiv 1$ on $I$, to a function $\eta: \tilde{I} \rightarrow \mathbb{R}$ by finding $\eta_{\ell} \in H^{m}\left(I_{\ell}\right), \eta_{r} \in H^{m}\left(I_{r}\right)$ with the boundary conditions (4.1), (4.2) given by

$$
\eta_{\ell}^{(k)}(a)=0 \text { for all } 0 \leq k \leq m-1, \quad \eta_{\ell}^{(k)}(b)=0 \text { for all } 1 \leq k \leq m-1, \eta_{\ell}(b)=1,
$$$$
\eta_{r}^{(k)}(d)=0 \text { for all } 0 \leq k \leq m-1, \quad \eta_{r}^{(k)}(c)=0 \text { for all } 1 \leq k \leq m-1, \eta_{r}(c)=1 .
$$

By Remark 2.2 the function $\eta:=E f=\chi_{I_{\ell}} \eta_{\ell}+\chi_{I} f+\chi_{I_{r}} \eta_{r}$ extended by 0 outside $\tilde{I}$ is then in $H^{m}(\mathbb{R})$ and satisfies (4.5). Due to the norm minimizing property of the extension operator $E$, the new function $\eta$ has minimal $H^{m}$-norm as well.

Lemma 4.4. For $a=-1, b=c=0, d=1, m \in \mathbb{N}$ arbitrary, we have constructed a cutoff function $\eta_{m} \in H^{m}(\mathbb{R})$ with $\operatorname{supp} \eta_{m}=[-1,1]$ satisfying $\eta_{m}(0)=1$ and $\eta_{m}^{(k)}(0)=0$ for all $1 \leq k \leq m-1$. These cutoff functions are displayed in Figure 3 for $m=1,2,3$.

4.3. Trace dependent operators and property (A). Next we discuss that trace dependent operators cannot be designed to satisfy (A). We recall that property (A) requires the existence of a continuous operator $A^{*}: H^{m}(\tilde{I}) \rightarrow H^{m}(I)$, i.e.

$$
\left\|A^{*} f\right\|_{H^{m}(I)} \lesssim\|f\|_{H^{m}(\tilde{I})} \quad \text { for all } f \in H^{m}(\tilde{I}),
$$

satisfying

$$
\langle A f, g\rangle_{L^{2}(\tilde{I})}=\left\langle f, A^{*} g\right\rangle_{L^{2}(I)} \quad \text { for all } f \in H^{m}(I), g \in H^{m}(\tilde{I}) .
$$

We first state a simple consequence of the properties (4.6) and (4.7). 


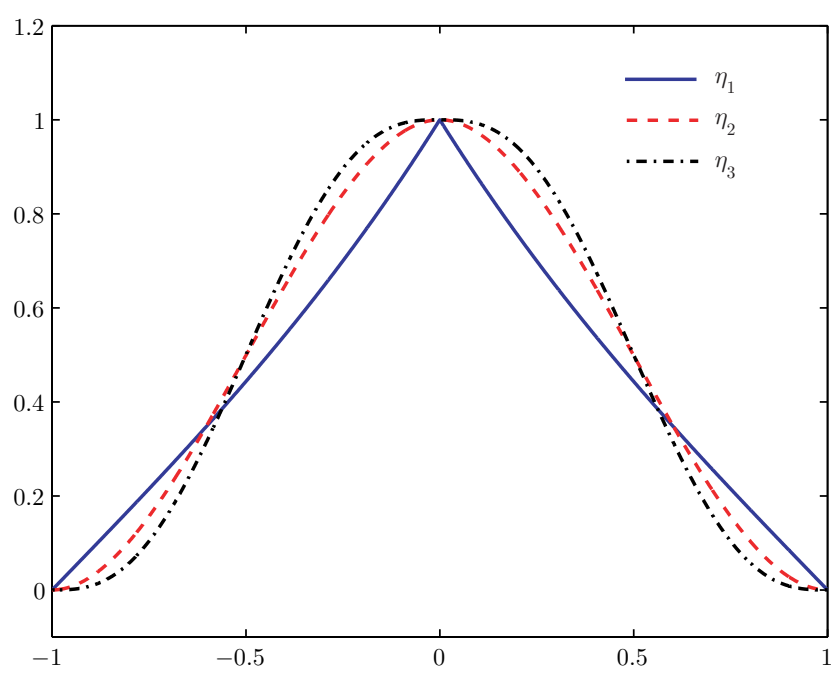

Figure 3. Cutoff functions $\eta_{m} \in H^{m}((-1,1))$ with minimal norm.

Lemma 4.5. Let (4.6) and (4.7) hold. Then for all $g \in H^{m}(\tilde{I})$ the functional

$$
A_{g}:=\langle A \cdot, g\rangle_{L^{2}(\tilde{I})}: H^{m}(I) \rightarrow \mathbb{R}
$$

is bounded in the $L^{2}$-norm, that is,

$$
\left|A_{g}(f)\right| \lesssim\|f\|_{L^{2}(I)} \quad \text { for all } f \in H^{m}(I) .
$$

By density of $H^{m}(I)$ in $L^{2}(I)$ this means in particular that $A_{g}$ can be extended uniquely to a functional

$$
A_{g}: L^{2}(I) \rightarrow \mathbb{R}, \text { and } A_{g} \in L^{2}(I)^{*} .
$$

Proof. By properties (4.6), (4.7), and the Cauchy-Schwartz inequality we have

$$
\begin{aligned}
\left|A_{g}(f)\right|=\left|\langle A f, g\rangle_{L^{2}(\tilde{I})}\right| & =\left|\left\langle f, A^{*} g\right\rangle_{L^{2}(I)}\right| \leq\|f\|_{L^{2}(I)}\left\|A^{*} g\right\|_{L^{2}(I)} \\
& \leq\|f\|_{L^{2}(I)}\left\|A^{*} g\right\|_{H^{m}(I)} \lesssim\|f\|_{L^{2}(I)}\|g\|_{H^{m}(\tilde{I})} .
\end{aligned}
$$

Thus, $A_{g}$ can be extended uniquely to $A_{g} \in L^{2}(I)^{*}$, and (4.8) follows.

The next result gives a first hint that (4.8) is not valid for trace dependent operators.

Lemma 4.6. Let $A: H^{m}(I) \rightarrow H^{m}(\tilde{I})$ be a trace dependent operator. Then $A$ cannot be extended to a continuous operator $A: L^{2}(I) \rightarrow L^{2}(\tilde{I})$. In particular, for every $m \in \mathbb{N}$ there exists a sequence $f_{k} \in H^{m}(I)$ with constant trace values such that $f_{k} \rightarrow 0$ in $L^{2}(I)$ and

$$
\frac{\left\|A f_{k}\right\|_{L^{2}(\tilde{I})}}{\left\|f_{k}\right\|_{L^{2}(I)}} \rightarrow \infty \quad \text { for } k \rightarrow \infty
$$




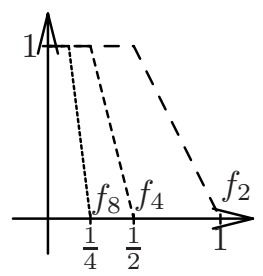

Figure 4. The functions $f_{k}$ defined in (4.9).

Proof. For convenience let $I=(0,1), \tilde{I}=(-1,2), I_{\ell}=(-1,0), I_{r}=(1,2)$. For $m=1$ and $k \in \mathbb{N}$ consider the sequence displayed in Figure 4,

$$
f_{k}(x)= \begin{cases}1, & x \in\left(0, \frac{1}{k}\right] \\ 2-k x, & x \in\left(\frac{1}{k}, \frac{2}{k}\right) \\ 0, & x \in\left[\frac{2}{k}, 1\right)\end{cases}
$$

Due to Remark 2.2, $f_{k} \in H^{1}(I)$ for all $k \in \mathbb{N}$ and $f_{k} \rightarrow 0$ in $L^{2}(I)$, as

$$
\int_{0}^{1}\left|f_{k}(x)\right|^{2} d x=\frac{1}{k}+\int_{\frac{1}{k}}^{\frac{2}{k}} k^{2}\left(\frac{2}{k}-x\right)^{2} d x=\frac{1}{k}-\left.\frac{k^{2}}{3}\left(\frac{2}{k}-x\right)^{3}\right|_{\frac{1}{k}} ^{\frac{2}{k}}=\frac{1}{k}+\frac{1}{3 k} \rightarrow 0
$$

for $k \rightarrow \infty$. As $A$ is trace dependent and $f_{k}(0)=1$ for all $k$, we have $\left.A f_{k}\right|_{I_{\ell}}=\left.A f_{\ell}\right|_{I_{\ell}}$ for all $k, \ell \in \mathbb{N}$, and as $A f \in H^{1}(\tilde{I})$, it is continuous. Thus $\left.A f_{k}\right|_{I_{\ell}} \not \equiv 0$ for all $k$, as $f_{k}(0)=1$ for all $k$. Thus, we have $\left\|A f_{k}\right\|_{L^{2}\left(I_{\ell}\right)}=\left\|A f_{\ell}\right\|_{L^{2}\left(I_{\ell}\right)}=: c>0$ for all $k, \ell \in \mathbb{N}$. Since $f_{k} \rightarrow 0$ in $L^{2}(I)$, we have

$$
\frac{\left\|A f_{k}\right\|_{L^{2}(\tilde{I})}}{\left\|f_{k}\right\|_{L^{2}(I)}} \geq \frac{\left\|A f_{k}\right\|_{L^{2}\left(I_{\ell}\right)}}{\left\|f_{k}\right\|_{L^{2}(I)}}=\frac{c}{\left\|f_{k}\right\|_{L^{2}(I)}} \rightarrow \infty \quad \text { for } k \rightarrow \infty .
$$

For larger $m$ we just have to make the sequence $f_{k}$ smoother in such a way that the boundary values remain unchanged, for instance by computing the $(m-1)$-fold convolution with $\chi_{\left[0, \frac{1}{2 k m}\right]}$, as this will result in convolutions in the desired space $H^{m}(I)$ with boundary values unchanged.

The necessary condition (4.8) from Lemma 4.5 now gives us a tool to prove the following main result.

Theorem 4.7. No trace dependent operator can fulfill (A).

Proof. Again, for convenience we choose $I=(0,1), \tilde{I}=(-1,2), I_{\ell}=(-1,0)$, $I_{r}=(1,2)$. We construct a function $g_{\varepsilon} \in H^{m}(\tilde{I})$ such that $A_{g_{\varepsilon}}$ is not continuous as a functional from $L^{2}$ to $\mathbb{R}$. This is a contradiction to (4.8) and thus a contradiction to (A). To this end, let $g_{\varepsilon} \in C^{\infty}(\tilde{I}), g_{\varepsilon} \geq 0$ and

$$
g_{\varepsilon}(x):= \begin{cases}0, & x \in(-1,-\varepsilon] \\ 1, & x \in\left[-\frac{\varepsilon}{2}, 2\right]\end{cases}
$$

Now we find $\varepsilon>0$ such that $A_{g_{\varepsilon}}=\left\langle A \cdot, g_{\varepsilon}\right\rangle_{L^{2}(\tilde{I})} \notin L^{2}(I)^{*}$. For $m \in \mathbb{N}$ let $\left\{f_{k}\right\}_{k} \subset$ $H^{m}(I)$ be the sequence introduced in the proof of Lemma 4.6. As stated there, we have $\left.A f_{k}\right|_{I_{\ell}}=\left.A f_{\ell}\right|_{I_{\ell}}$ for all $k, \ell \in \mathbb{N}$ and $A f_{k}(0)=1$. Since $A f_{k} \in H^{m}(\tilde{I}), A f_{k}$ is continuous. Thus, there exists $\delta>0$ not depending on $k$ such that $A f_{k}(x)>\frac{1}{2}$ for 
all $k$ and all $x \in[-\delta, 0]$. Choose $\varepsilon:=\delta$ and $g_{\varepsilon}$ as defined above. As $f_{k} \geq 0, g_{\varepsilon} \geq 0$ we then have

$$
\frac{\left|\left\langle A f_{k}, g_{\varepsilon}\right\rangle_{L^{2}(\tilde{I})}\right|}{\left\|f_{k}\right\|_{L^{2}(I)}} \geq \frac{\left\langle A f_{k}, g_{\varepsilon}\right\rangle_{L^{2}\left(I_{\ell}\right)}}{\left\|f_{k}\right\|_{L^{2}(I)}} \geq \frac{\frac{1}{2} \cdot \frac{\delta}{2}}{\left\|f_{k}\right\|_{L^{2}(I)}}=\frac{\delta}{4\left\|f_{k}\right\|_{L^{2}(I)}} \rightarrow \infty,
$$

as $f_{k} \rightarrow 0$ in $L^{2}(I)$. This means $A_{g_{\varepsilon}} \notin L^{2}(I)^{*}$, which is the desired contradiction.

\section{WORKING AROUND THE TRACE}

Although extension operators which interpolate trace values do not explicitly satisfy (A), they will lead us to deduce an extension operator in Definition 5.3 which turns out to be similar to the Hestenes extension used in DS. To this end, we concentrate on extending functions $f \in H^{m}(0, \infty)$ to $A f \in H^{m}(\mathbb{R})$. Two-sided extensions are then a simple modification of such an operator. The crucial point of the extension process is the satisfaction of an interpolation property like (4.1) of $A f$ at $x=0$ on the one hand and continuity of $A$ and $A^{*}$ on the other hand. First we focus on the interpolation property. What we are looking for is an operator $A$ which satisfies the interpolation property

$$
\left.(A f)^{(k)}(x)\right|_{x=0-}=f^{(k)}(0), \quad \text { for } k=0, \ldots, m-1,
$$

together with $\left.A f\right|_{(-\infty, 0)} \in H^{m}((-\infty, 0))$. Consider the Taylor polynomial $T_{x_{0}}$ of $f$ at $x_{0}=0$ from Subsection 4.1. Obviously,

$$
\left(T_{x_{0}} f\right)(x)=\sum_{j=0}^{m-1} \frac{f^{(j)}\left(x_{0}\right)}{j !}\left(x-x_{0}\right)^{j}=\sum_{j=0}^{m-1} \frac{f^{(j)}(0)}{j !} x^{j}
$$

satisfies the interpolation property (5.1). Recall that the operator $E_{T}$ in Subsection 4.1 was built in terms of $T_{x_{0}}$. We now aim at deriving an approximation of the Taylor polynomial which is not trace dependent, employing divided differences; see, e.g., Bo] for details.

Definition 5.1. Let $I \subset \mathbb{R}$ be an interval, $f: I \rightarrow \mathbb{R}$ and $\beta_{j} \in I, j=1, \ldots, k$, be pairwise distinct. Then the divided differences $\left[\beta_{1}, \ldots, \beta_{k}\right]_{f}$ of $f$ are defined as

$$
\begin{aligned}
{\left[\beta_{1}\right]_{f} } & :=f\left(\beta_{1}\right), \\
{\left[\beta_{1}, \ldots, \beta_{k}\right]_{f} } & :=\frac{\left[\beta_{2}, \ldots, \beta_{k}\right]_{f}-\left[\beta_{1}, \ldots, \beta_{k-1}\right]_{f}}{\beta_{k}-\beta_{1}} .
\end{aligned}
$$

For $I$ open and $f \in C^{k}(I)$, the divided differences satisfy

$$
\left[\beta_{1}, \ldots, \beta_{k}\right]_{f} \rightarrow \frac{f^{(k-1)}\left(x_{0}\right)}{(k-1) !}, \quad \text { if } \beta_{j} \rightarrow x_{0} \text { for } j=1, \ldots, k .
$$

Hence for $f \in C^{k}(I),\left[\beta_{1}, \ldots, \beta_{k}\right]_{f}$ can be extended to a continuous function $\left[\beta_{1}, \ldots, \beta_{k}\right]_{f}: I \times \cdots \times I \rightarrow \mathbb{R}$. Due to (5.4), $T_{x_{0}}$ from (5.2) can also be written as

$$
\left(T_{x_{0}} f\right)(x)=(N f)(x):=\sum_{j=1}^{m}\left[\beta_{1}, \ldots, \beta_{j}\right]_{f} \prod_{i=1}^{j}\left(x-\beta_{i}\right)=\sum_{j=1}^{m}\left[\beta_{1}, \ldots, \beta_{j}\right]_{f} x^{j-1} .
$$


Note that we cannot compute the incorporated divided differences explicitly as long as some $\beta_{j}$ coincide. This leads us to the following approach. For $\beta_{j}>0$ pairwise distinct, find a polynomial $P_{\beta} f$ solving the interpolation problem

$$
\left(P_{\beta} f\right)\left(\beta_{j}\right)=f\left(\beta_{j}\right), \quad j=1, \ldots, m .
$$

The solution polynomial can be written in Newton form as

$$
\left(P_{\beta} f\right)(x)=\sum_{j=1}^{m}\left[\beta_{1}, \ldots, \beta_{j}\right]_{f} \prod_{i=1}^{j}\left(x-\beta_{i}\right) .
$$

Because of (5.4) we have pointwise convergence,

$$
\left(P_{\beta} f\right)(x) \rightarrow(N f)(x), \quad \text { for all } x, \text { if } \beta_{j} \rightarrow 0 \text { for all } j=1, \ldots, m .
$$

Hence for small $\beta_{j}$, the polynomial $P_{\beta}$ can be regarded as a good approximation of the Taylor polynomial in (5.2). Of course, it still does not satisfy the interpolation property (5.1). We now aim at building the convergence of the $\beta_{j}$ into the interpolating polynomial $P_{\beta}$. Clearly we have

$$
\beta_{j} x \rightarrow 0 \quad \text { for all } j=1, \ldots, m \text { if } x \rightarrow 0 .
$$

So by (5.6) the convergence $\left(P_{\beta x} f\right)(y) \rightarrow(N f)(y)$ for $x \rightarrow 0$ holds for all $y$. As we are just interested in an approximation of the Taylor polynomial $\left(T_{0} f\right)(x)$ for $x$ close to 0 , we expect $\left(P_{\beta x} f\right)(x)$ to satisfy the interpolation property (5.1). Before we establish this property in Proposition 5.2, we first find a simple representation for $\left(P_{\beta x} f\right)(x)$. We have

$$
\begin{aligned}
\left(P_{\beta x} f\right)(x) & =\sum_{j=1}^{m}\left[\beta_{1} x, \ldots, \beta_{j} x\right]_{f} \prod_{i=1}^{j}\left(x-\beta_{i} x\right) \\
& =\sum_{j=1}^{m} f\left(\beta_{j} x\right) \prod_{i=1, i \neq j}^{m} \frac{x-\beta_{j} x}{\beta_{j} x-\beta_{i} x}=\sum_{j=1}^{m} f\left(\beta_{j} x\right) \prod_{i=1, i \neq j}^{m} \frac{1-\beta_{i}}{\beta_{j}-\beta_{i}} .
\end{aligned}
$$

Here we have switched in the second identity to the Lagrange representation of the interpolation polynomial $P_{\beta x} f$. Now we are ready to define an operator which satisfies the interpolation property (5.1) which is not an extension operator yet, as it neither extends $f$ to the negative range nor leaves $f$ unchanged on its range.

Proposition 5.2. For $\beta_{j}>0$ for $j=1, \ldots, m$ and $x>0$, define $I_{H}: H^{m}\left(\mathbb{R}^{+}\right) \rightarrow$ $H^{m}\left(\mathbb{R}^{+}\right)$by

$$
\left(I_{H} f\right)(x):=\left(P_{\beta x} f\right)(x)=\sum_{j=1}^{m} f\left(\beta_{j} x\right) \prod_{i=1, i \neq j}^{m} \frac{1-\beta_{i}}{\beta_{j}-\beta_{i}} .
$$

Then $I_{H}$ is an interpolation operator satisfying the interpolation property (5.1).

Proof. Obviously, $I_{H} f \in H^{m}\left(\mathbb{R}^{+}\right)$if $f \in H^{m}\left(\mathbb{R}^{+}\right)$. We want to prove

$$
\left.\left(I_{H} f\right)^{(k)}(x)\right|_{x=0}=f^{(k)}(0) \quad \text { for } k=0, \ldots, m-1 .
$$


We have for all $k \leq m-1$

$$
\begin{aligned}
\left.\left(I_{H} f\right)^{(k)}(x)\right|_{x=0} & =\left.\sum_{j=1}^{m} f^{(k)}\left(\beta_{j} x\right) \beta_{j}^{k} \prod_{i \neq j}^{m} \frac{1-\beta_{i}}{\beta_{j}-\beta_{i}}\right|_{x=0} \\
& =f^{(k)}(0) \sum_{j=1}^{m} \beta_{j}^{k} \prod_{i \neq j}^{m} \frac{1-\beta_{i}}{\beta_{j}-\beta_{i}}=f^{(k)}(0)
\end{aligned}
$$

because of the identity

$$
\sum_{j=1}^{m} \beta_{j}^{k} \prod_{i \neq j}^{m} \frac{1-\beta_{i}}{\beta_{j}-\beta_{i}}=1
$$

in the last equation. To see this, we claim that

$$
\sum_{j=1}^{m} \beta_{j}^{k} \prod_{i \neq j}^{m} \frac{x-\beta_{i}}{\beta_{j}-\beta_{i}}=x^{k}
$$

holds for all $x \in \mathbb{R}$ and $0 \leq k \leq m-1$. Indeed, both sides of the equation are polynomials of degree $\leq m-1$ which coincide on the $m$ sampling points $\beta_{j}$. Hence they are equal. Applying this to $x=1$ yields the desired identity.

By a modification we obtain an extension operator.

Proposition 5.3. For $f \in H^{m}\left(\mathbb{R}^{+}\right), \beta_{j}<0, j=1, \ldots, m$, define $E_{H}: H^{m}\left(\mathbb{R}^{+}\right) \rightarrow$ $H^{m}(\mathbb{R})$ by

$$
\left(E_{H} f\right)(x):=\chi_{\mathbb{R}^{+}}(x) f(x)+\chi_{(-\infty, 0)} \sum_{j=1}^{m} f\left(\beta_{j} x\right) \prod_{i=1, i \neq j}^{m} \frac{1-\beta_{i}}{\beta_{j}-\beta_{i}} .
$$

Then $E_{H}$ is an extension operator satisfying the interpolation property (5.1).

Proof. It is clear from the proof of Proposition 5.2 that $E_{H} f$ satisfies the interpolation property (5.1). To prove $E_{H} f \in H^{m}(\mathbb{R})$ we remark that $\left.E_{H} f\right|_{(-\infty, 0)} \in$ $H^{m}((-\infty, 0))$. Together with the interpolation property (5.1) and the gluing behavior stated in Remark 2.2] we have $E_{H} f \in H^{m}(\mathbb{R})$.

Note that in (5.7) the $\beta_{j}$ are all negative now, ensuring that $f$ is evaluated in its parameter domain $\mathbb{R}^{+}$in the second term. The definition in Proposition 5.3 can be generalized to some extent. Revisiting the crucial step in the proof of smoothness in Proposition 5.2, we recall that smoothness was obtained since for $\ell=m$ we had

$$
\sum_{j=0}^{\ell} \beta_{j}^{k} \prod_{i \neq j}^{\ell} \frac{1-\beta_{i}}{\beta_{j}-\beta_{i}}=: \sum_{j=0}^{\ell} \beta_{j}^{k} \alpha_{j}=1, \quad \text { for } k=0, \ldots, m-1 .
$$

This is a Vandermonde system for the $\alpha_{j}$. Thus, it is ill conditioned for the $\beta_{j}$ being close to each other which results in a solution vector $\alpha$ with a large Euclidean norm $\|\alpha\|_{2}$. If we now allow $\ell$ to become greater than $m$ and still require the $\ell$ sampling points $\beta_{j}$ to satisfy (5.8), we observe smaller solutions $\alpha$ measured in $\|\cdot\|_{2}$, solving the system (5.8) by the least squares method. Although in the course of the optimization process we will realize that the operator norm of $E_{H}$ does not decrease when the number $\ell$ of sampling points is increased, we restate the definition in Proposition 5.3 for this setting. 
Proposition 5.4. Given the smoothness level $m$, let $\ell \geq m$. Let the numbers

$$
\beta_{1}, \ldots, \beta_{\ell}<0 \text { pairwise distinct, } \quad \alpha_{1}, \ldots, \alpha_{\ell},
$$

solve the linear Vandermonde system $\sum_{j=1}^{\ell} \alpha_{j} \beta_{j}^{k}=1, k=0, \ldots, m-1$. Then the operator $\tilde{E}_{H}$, defined by $\left(\tilde{E}_{H} f\right)(x):=\chi_{\mathbb{R}^{+}}(x) f(x)+\chi_{(-\infty, 0)} \sum_{j=1}^{\ell} \alpha_{j} f\left(\beta_{j} x\right)$, is an extension operator $E: H^{m}\left(\mathbb{R}^{+}\right) \rightarrow H^{m}(\mathbb{R})$ satisfying the interpolation property (5.1).

The proof is the same as in Proposition 5.3. Although $\tilde{E}_{H}$ is very similar to the Hestenes extension introduced in [DS], it still suffers from the following deficiencies. First, $\tilde{E}_{H}$ extends functions in $H^{m}\left(\mathbb{R}^{+}\right)$to functions in $H^{m}(\mathbb{R})$ rather than functions in $H^{m}(I)$ to functions in $H^{m}(\tilde{I})$. Second, in (A) we require zero boundary values. Third, $\tilde{E}_{H}$ is an extension operator on the primal side, but we do not yet have any grip on the adjoint $\tilde{E}_{H}^{*}$ relative to the $L_{2}$ inner product. Further we need to modify $\tilde{E}_{H}$ to finally obtain an operator satisfying (A).

\section{The Hestenes extension}

Given the smoothness level $m$, let $\ell \geq 2 m$. Let real numbers

$$
\beta_{1}, \ldots, \beta_{\ell}<0 \text { pairwise distinct, } \quad \alpha_{1}, \ldots, \alpha_{\ell},
$$

solve the linear Vandermonde system

$$
\sum_{j=1}^{\ell} \alpha_{j} \beta_{j}^{k}=1, \quad k=-m, \ldots, m-1 .
$$

Defining

$$
V_{m}:=\left(\begin{array}{ccc}
\beta_{1}^{-m} & \cdots & \beta_{\ell}^{-m} \\
\vdots & \ddots & \vdots \\
\beta_{1}^{m-1} & \cdots & \beta_{\ell}^{m-1}
\end{array}\right), \quad b:=\left(\begin{array}{c}
1 \\
\vdots \\
1
\end{array}\right),
$$

this can be written as the linear system of equations

$$
V_{m} \alpha=b
$$

for $\alpha:=\left(\alpha_{1}, \ldots, \alpha_{\ell}\right)^{T} \in \mathbb{R}^{\ell}$. We can assume without loss of generality that the $\beta_{j}$ are sorted such that

$$
\min _{j} \beta_{j}=\beta_{1}<\cdots<\beta_{\ell}=\max _{j} \beta_{j}<0 .
$$

Remark 6.1. 1. Obviously, $\ell=2 m$ suffices to obtain a unique solution of (6.4). For greater $\ell$ smaller solutions $\alpha$, with respect to the Euclidean $\|\cdot\|_{2}$ norm, may be obtained by the least squares approach

$$
\alpha=V_{m}^{T}\left(V_{m} V_{m}^{T}\right)^{-1} b,
$$

which is the smallest solution of (6.4) with respect to $\|\cdot\|_{2}$. We will see in Subsection 7.3 that $\ell=2 m$ is actually the best choice.

2. In [DS] it was assumed that $\ell \geq 2 m+2$ and that the $\beta_{j}, \alpha_{j}$ satisfy (6.2) in the larger range $k=-m-1, \ldots, m$. This is necessary for smooth extensions in the $C^{m}$-sense. However, as we are indeed interested in smooth extensions in the $H^{m}$-sense, by Remark $2.22 m$ conditions suffice. In fact, this observation will mean a great improvement to the condition of the Hestenes extension. 


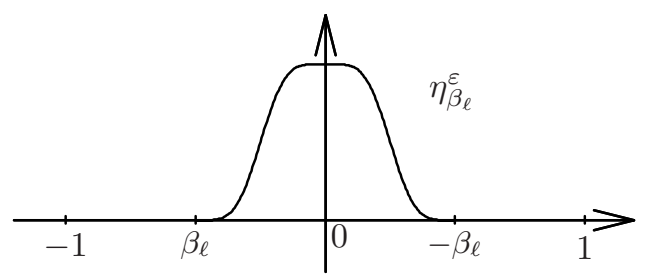

FIGURE 5. The function $\eta_{\beta_{\ell}}^{\varepsilon}$ with $\varepsilon=1$.

Additionally, we need a cutoff function $\eta_{\beta_{\ell}}^{\varepsilon} \in H^{m}(\mathbb{R})$, where $\varepsilon \in(0,1]$ and $\beta_{\ell}$ is the largest sampling point in (6.5). Let

$$
\eta_{\beta_{\ell}}^{\varepsilon}(x):=\eta_{m}\left(\frac{x}{-\beta_{\ell} \varepsilon}\right)
$$

where $\eta_{m}$ is the cutoff function defined in Lemma 4.4. We see in Figure 5 that as long as $\beta_{\ell}>-1, \eta_{\beta_{\ell}}^{\varepsilon}$ satisfies the support property

$$
\operatorname{supp} \eta_{\beta_{\ell}}^{\varepsilon} \subset\left(\left[\varepsilon \beta_{\ell},-\varepsilon \beta_{\ell}\right] \cap[-\varepsilon, \varepsilon]\right)
$$

together with the growth property at $x=0$,

$$
\eta_{\beta_{\ell}}^{\varepsilon}(0)=1, \quad \eta_{\beta_{\ell}}^{\varepsilon(k)}(0)=0 \quad \text { for } k=1, \ldots, m-1 .
$$

In DS] $\beta_{1}$ was bounded by -2 from below, which is indeed not necessary after specifying the cutoff function by (6.7). These prerequisites at hand, we can now state

Definition 6.2. Let $I=(0,1), \tilde{I}=(-1,2), I_{\ell}=(-1,0), I_{r}=(1,2)$. The Hestenes extension $A: H^{m}(I) \rightarrow H^{m}(\tilde{I})$ of $f$ is defined by

$$
\begin{aligned}
(A f)(x):= & \chi_{I}(x) f(x)+\chi_{I_{\ell}}(x) \sum_{j=1}^{\ell} \alpha_{j}\left(\eta_{\beta_{\ell}}^{\varepsilon} f\right)\left(\beta_{j} x\right) \\
& +\chi_{I_{r}}(x) \sum_{j=1}^{\ell} \alpha_{j} \eta_{\beta_{\ell}}^{\varepsilon}\left(\beta_{j}(x-1)\right) f\left(1+\beta_{j}(x-1)\right) .
\end{aligned}
$$

Its adjoint $A^{*}: H^{m}(\tilde{I}) \rightarrow H^{m}(I)$ of $A$ relative to the $L^{2}$ inner product can be computed as

$$
\begin{aligned}
A^{*} g= & \chi_{I}(x) g(x)-\chi_{I_{\ell}}(x-1) \sum_{j=1}^{\ell} \beta_{j}^{-1} \alpha_{j} \eta_{\beta_{\ell}}^{\varepsilon}(x) g\left(\beta_{j}^{-1} x\right) \\
& -\chi_{I_{r}}(1+x) \sum_{j=1}^{\ell} \beta_{j}^{-1} \alpha_{j} \eta_{\beta_{\ell}}^{\varepsilon}(x-1) g\left(1+\beta_{j}^{-1}(x-1)\right) .
\end{aligned}
$$

Lemma 6.3. The Hestenes extension A defined in (6.2) satisfies (A). Furthermore, we have supp $A f \subset[-\varepsilon, 1+\varepsilon]$.

Proof. We have to show (L), $(\mathrm{CA}),\left(\mathrm{CA}^{*}\right)$ and that $A$ is well defined; i.e., $A f \in$ $H^{m}(\tilde{I})$ for all $f \in H^{m}(I)$. (CA) is proved in Lemma 6.4 below. The proof of $\left(\mathrm{CA}^{*}\right)$ is analogous in view of (6.11). We start by proving that $A$ maps $H^{m}$-functions to $H^{m}$-functions. Due to the product rule for $H^{m}$-functions we certainly have $\left.A f\right|_{I_{\ell}} \in H^{m}\left(I_{\ell}\right),\left.A f\right|_{I}=f \in H^{m}(I),\left.A f\right|_{I_{r}} \in H^{m}\left(I_{r}\right)$. In view of Remark 2.2 we 
have to check whether the first $m-1$ trace values of $\left.A f\right|_{I_{\ell}}$ and $f$ coincide at $x=0$, and of $\left.A f\right|_{I_{r}}$ and $f$ at $x=1$, respectively. As $f, \eta_{\beta_{\ell}}^{\varepsilon} \in C^{m-1}(\bar{I})$ by Theorem 2.1, we can differentiate (6.10) from the left at $x=0$ using the Leibniz rule and compute for $k=0, \ldots, m-1$

$$
\begin{aligned}
(A f)^{(k)}(0) & =\sum_{j=1}^{\ell} \alpha_{j} \beta_{j}^{k}\left(\eta_{\beta_{\ell}}^{\varepsilon} f\right)^{(k)}(0)=\sum_{j=1}^{\ell} \alpha_{j} \beta_{j}^{k} \sum_{i=0}^{k}\left(\begin{array}{c}
k \\
i
\end{array}\right) \eta_{\beta_{\ell}}^{\varepsilon(k-i)}(0) f^{(i)}(0) \\
& =\sum_{j=1}^{\ell} \alpha_{j} \beta_{j}^{k} \eta_{\beta_{\ell}}^{\varepsilon}(0) f^{(k)}(0)=f^{(k)}(0)
\end{aligned}
$$

due to (6.2) and the behaviour of $\eta_{\beta_{\ell}}^{\varepsilon}$ at $x=0$ described in (6.9). Differentiating (6.10) from the right at $x=1$ analogously yields

$$
(A f)^{(k)}(1)=\sum_{j=1}^{\ell} \alpha_{j} \beta_{j}^{k} \eta(0) f^{(k)}(1)=f^{(k)}(1), \quad k=0, \ldots, m .
$$

Similarly, we get $A^{*}: H^{m}(\tilde{I}) \rightarrow H_{0}^{m}(I)$, now using (6.2) for $k=-m, \ldots,-1$. To see that $A^{*}$ from (6.11) really coincides with the $L^{2}$-adjoint of $A$, we compute for $f \in L^{2}(I), g \in L^{2}(\tilde{I})$

$$
\begin{aligned}
\int_{\tilde{I}} A f g d x= & \int_{[0,1]} f(x) g(x) d x+\int_{[-1,0]} \sum_{j=1}^{\ell} \alpha_{j}\left(\eta_{\beta_{\ell}}^{\varepsilon} f\right)\left(\beta_{j} x\right) g(x) d x \\
& +\sum_{j=1}^{\ell} \int_{[1,2]} \alpha_{j} \eta_{\beta_{\ell}}^{\varepsilon}\left(\beta_{j}(x-1)\right) f\left(1+\beta_{j}(x-1)\right) g(x) d x \\
= & \int_{[0,1]} f(x) g(x) d x-\sum_{j=1}^{\ell} \int_{\left[0,-\beta_{j}\right]} \beta_{j}^{-1} \alpha_{j}\left(\eta_{\beta_{\ell}}^{\varepsilon} f\right)(x) g\left(\beta_{j}^{-1} x\right) d x \\
& -\sum_{j=1}^{\ell} \int_{\left[1+\beta_{j}, 1\right]} \beta_{j}^{-1} \alpha_{j} \eta_{\beta_{\ell}}^{\varepsilon}(x-1) f(x) g\left(\beta_{j}^{-1}(x-1)+1\right) d x \\
= & \int_{[0,1]} f(x) g(x) d x-\int_{[0,1]} \sum_{j=1}^{\ell} \beta_{j}^{-1} \alpha_{j}\left(\eta_{\beta_{\ell}}^{\varepsilon} f\right)(x) g\left(\beta_{j}^{-1} x\right) d x \\
& -\int_{[0,1]} \sum_{j=1}^{\ell} \beta_{j}^{-1} \alpha_{j} \eta_{\beta_{\ell}}^{\varepsilon}(x-1) f(x) g\left(\beta_{j}^{-1}(x-1)+1\right) d x .
\end{aligned}
$$

Here we simply substituted $x \mapsto \beta_{j}^{-1} x$ in the second integral and $x \mapsto \beta_{j}^{-1}(x-1)+1$ in the third integral. We were allowed to extend the integral domains as we have $\eta_{\beta_{\ell}}^{\varepsilon}(x)=0$ for $x>-\beta_{j}$ and $\eta_{\beta_{\ell}}^{\varepsilon}(x-1)=0$ for $x<1+\beta_{j}$ due to (6.8), bearing in mind that $\beta_{j} \leq \beta_{\ell}$ for all $j$.

We are left with showing (L); i.e., we have to prove zero boundary values for the extension $A f$. We will prove that $\operatorname{supp} A f \subset[-\varepsilon, 1+\varepsilon]$ and that $A f$ has zero boundary values in its support. For the support, note that obviously

$$
\operatorname{supp} A f \subset \operatorname{supp} \sum_{j=1}^{\ell} \eta_{\beta_{\ell}}^{\varepsilon}\left(\beta_{j} x\right) \subset \bigcup_{j=1}^{\ell} \operatorname{supp} \eta_{\beta_{\ell}}^{\varepsilon}\left(\beta_{j} x\right) \subset \operatorname{supp} \eta_{\beta_{\ell}}^{\varepsilon}\left(\beta_{\ell} x\right)
$$


because $\beta_{j} \leq \beta_{\ell}$ and

$$
\operatorname{supp} \eta_{\beta_{\ell}}^{\varepsilon}\left(\beta_{\ell} x\right)=\operatorname{supp} \eta_{m}\left(\frac{x}{\varepsilon}\right)=[-\varepsilon, 1+\varepsilon]
$$

by the definition of $\eta_{\beta_{\ell}}^{\varepsilon}$ in (6.7) and the cutoff function $\eta_{m}$ from Lemma 4.4 As $f\left(\beta_{j} x\right)$ is bounded and $\eta_{\beta_{\ell}}^{\varepsilon}\left(\beta_{j} x\right)$ has zero boundary values by Lemma 4.4 in its support $\operatorname{supp} \eta_{\beta_{\ell}}^{\varepsilon}\left(\beta_{j} x\right)=\left[\beta_{j} \varepsilon,-\beta_{j} \varepsilon\right]$ (see (6.8)), it is clear that $A f$ also has zero boundary values in its support $[-\varepsilon, 1+\varepsilon]$, as $A f$ is just a sum of the $\eta_{\beta_{\ell}}^{\varepsilon}$ weighted with bounded functions. Finally note that although $f$ and $g$ might be evaluated outside their domains $I, \tilde{I}$, respectively, in the definitions of $A$ and $A^{*}$ the operators are still well defined as in those cases the support of the cutoff function $\eta_{\beta_{\ell}}^{\varepsilon}$ ensures that the corresponding term is annihilated.

We will use an estimate of the operator norm of $A$ to further optimize the parameters involved.

Lemma 6.4. Let $m \geq 1$ and $\eta_{m}$ be as given in Lemma 4.4. In view of (6.5), we have the estimate

$$
\|A f\|_{H^{m}(\tilde{I})} \leq\left(1+2 \sqrt{\ell} \frac{\left(-\beta_{1}\right)^{m-\frac{1}{2}}}{\left(-\beta_{\ell}\right)^{m}}\|\alpha\|_{2}\left\|\eta_{m}\right\|_{W^{m, \infty}(\mathbb{R})}\right)\|f\|_{H^{m}(I)} .
$$

Proof. From (6.10) we obtain by the triangle inequality

$$
\begin{aligned}
\|A f\|_{H^{m}(\tilde{I})} \leq & \|f\|_{H^{m}(I)}+\left\|\sum_{j=1}^{\ell} \alpha_{j}\left(\eta_{\beta_{\ell}}^{\varepsilon} f\right)\left(\beta_{j} x\right)\right\|_{H^{m}\left(I_{\ell}\right)} \\
& +\left\|\sum_{j=1}^{\ell} \alpha_{j} \eta_{\beta_{\ell}}^{\varepsilon}\left(\beta_{j}(x-1)\right) f\left(1+\beta_{j}(x-1)\right)\right\|_{H^{m}\left(I_{r}\right)} .
\end{aligned}
$$

We just prove the inequality for the second term; for the third it is analogous:

$$
\begin{aligned}
\left\|\sum_{j=1}^{\ell} \alpha_{j}\left(\eta_{\beta_{\ell}}^{\varepsilon} f\right)\left(\beta_{j} x\right)\right\|_{H^{m}\left(I_{\ell}\right)} & \leq \sum_{j=1}^{\ell}\left|\alpha_{j}\right|\left\|\left(\eta_{\beta_{\ell}}^{\varepsilon} f\right)\left(\beta_{j} x\right)\right\|_{H^{m}\left(I_{\ell}\right)} \\
& \leq\left(\sum_{j=1}^{\ell}\left|\alpha_{j}\right|\left|\frac{\beta_{j}}{\beta_{\ell}}\right|^{m-\frac{1}{2}}\right)\left\|\eta_{m}(x) f\left(\beta_{\ell} x\right)\right\|_{H^{m}(I)} \\
& \lesssim\left(\sum_{j=1}^{\ell}\left|\alpha_{j}\right|\left|\beta_{j}\right|^{m-\frac{1}{2}}\right) \frac{\left\|\eta_{m}\right\|_{W^{m, \infty}(\mathbb{R})}}{\left|\beta_{\ell}\right|^{m}}\|f\|_{H^{m}(I)}
\end{aligned}
$$

Here we have used twice that for any $\varphi \in H^{m}$ and any $\lambda \in \mathbb{R}$ we have $\|\varphi(\lambda x)\|_{H^{m}} \leq$ $|\lambda|^{-\frac{1}{2}} \cdot \max \left\{1,|\lambda|^{m}\right\}\|\varphi\|_{H^{m}}$. We can now apply various finite dimensional Hölder 
inequalities to the sum above. Using $\left\|\left(a_{j} b_{j}\right)_{j}\right\|_{1} \leq\left\|\left(a_{j}\right)_{j}\right\|_{2}\left\|\left(b_{j}\right)_{j}\right\|_{2}$, we can estimate

$$
\begin{aligned}
\frac{\left\|\eta_{m}\right\|_{W^{m, \infty}(\mathbb{R})}}{\left|\beta_{\ell}\right|^{m}}\|f\|_{H^{m}(I)} & \sum_{j=1}^{\ell}\left|\alpha_{j}\right|\left|\beta_{j}\right|^{m-\frac{1}{2}} \\
& \leq\|\alpha\|_{2}\left\|\left(\left|\beta_{j}\right|^{m-\frac{1}{2}}\right)_{j}\right\|_{2} \frac{\left\|\eta_{m}\right\|_{W^{m, \infty}(\mathbb{R})}}{\left|\beta_{\ell}\right|^{m}}\|f\|_{H^{m}(I)} \\
& \leq \sqrt{\ell} \frac{\left(-\beta_{1}\right)^{m-\frac{1}{2}}}{\left(-\beta_{\ell}\right)^{m}}\|\alpha\|_{2}\left\|\eta_{m}\right\|_{W^{m, \infty}(\mathbb{R})} .
\end{aligned}
$$

\section{Optimization of the Hestenes extension}

We now use the estimate (6.12) for optimizing the operator norm of $A$; i.e., we minimize the functional

$$
F\left(\beta_{1}, \ldots, \beta_{\ell}\right):=\ell \frac{\left(-\beta_{1}\right)^{2 m-1}}{\left(-\beta_{\ell}\right)^{2 m}}\|\alpha\|_{2}^{2}=\ell \frac{\left(-\beta_{1}\right)^{2 m-1}}{\left(-\beta_{\ell}\right)^{2 m}} \sum_{j=1}^{\ell} \alpha_{j}\left(\beta_{1}, \ldots, \beta_{\ell}\right)^{2} .
$$

We will later apply a gradient method to $F$. For this to be practicable, we first deduce for $\ell=2 m$ an explicit representation for the $\alpha_{j}$ in terms of the $\beta_{j}$.

7.1. Optimization for $\ell=2 m$.

Lemma 7.1. The solution $\alpha$ of the Vandermonde system (6.2) for the special case $\ell=2 m$ is given by

$$
\alpha_{j}=\beta_{j}^{m} \prod_{i=1, i \neq j}^{\ell}\left(\frac{1-\beta_{i}}{\beta_{j}-\beta_{i}}\right)
$$

with partial derivatives

$$
\partial_{\beta_{k}} \alpha_{j}= \begin{cases}\alpha_{j} \frac{1-\beta_{j}}{\left(1-\beta_{k}\right)\left(\beta_{j}-\beta_{k}\right)}, & \text { if } k \neq j, \\ \alpha_{j}\left(\frac{m}{\beta_{j}}-\sum_{i=1, i \neq j}^{2 m}\left(\beta_{j}-\beta_{i}\right)^{-1}\right), & \text { if } k=j .\end{cases}
$$

Proof. Applying Cramer's rule to the matrix system (6.4) we find for the solutions $\alpha_{j}$

$$
\alpha_{j}=\frac{\left|\begin{array}{ccccccc}
\beta_{1}^{-m} & \cdots & \beta_{j-1}^{-m} & 1 & \beta_{j+1}^{-m} & \cdots & \beta_{\ell}^{-m} \\
\vdots & & \vdots & \vdots & \vdots & & \vdots \\
\beta_{1}^{m-1} & \cdots & \beta_{j-1}^{m-1} & 1 & \beta_{j+1}^{m-1} & \cdots & \beta_{\ell}^{m-1}
\end{array}\right|}{\left|V_{m}\right|}
$$

where $|M|:=\operatorname{det} M$. For the Vandermonde determinant we have

$$
\left|V_{m}\right|=\prod_{i=1}^{\ell} \beta_{i}^{-m} \prod_{i<j}^{\ell}\left(\beta_{j}-\beta_{i}\right) .
$$

It remains to deduce a formula for the determinant in the numerator in (7.4). Note that the matrix in the numerator has been derived by simply filling the $j$-th column with 1's, or, equivalently, replacing the real number $\beta_{j}$ by 1 . So we obtain a modified 
Vandermonde system with the determinant given by (7.5) with $\beta_{j}$ replaced by 1 . Consequently, combining (7.4), (7.5), and this last observation, we get

$$
\alpha_{j}=\beta_{j}^{m} \prod_{i=1, i \neq j}^{\ell}\left(\frac{1-\beta_{i}}{\beta_{j}-\beta_{i}}\right) .
$$

Straightforward computations then yield (7.3).

Note that we could have also followed the lines of the proof after Proposition 5.2 to prove the explicit representation (7.2). We now use the explicit representation of the $\alpha_{j}(7.2)$ to minimize the functional $F$ in (7.1) by a gradient projection method described in [B]; i.e., $F$ is being minimized successively by descending along the gradient of $F$ projected onto the linear admissible range given by (6.1).

7.2. Numerical results of the optimization process for $\ell=2 \mathrm{~m}$. Comparing our optimized Hestenes extension with the operator found in [DS], two relaxations are made here. First, $\ell \geq 2 m$ of the $\beta_{j}$ 's suffice, whereas in [DS], $\ell \geq 2 m+2$ of the $\beta_{j}$ 's were used to satisfy (6.2) in the larger range $k=-m-1, \ldots, m$; see Remark 6.1.2. Second, by adapting the support of the cutoff function $\eta_{\beta_{\ell}}^{\varepsilon}$ in (6.8), it turns out that the restriction of the $\beta_{j}$ from above and below (i.e., $\left.-2 \leq \beta_{1}<\cdots<\beta_{\ell} \leq-\frac{1}{2}\right)$ is actually not necessary.

Apart from these changes, the main ingredient of the above optimization process is to find the optimal distribution of the $\beta_{j}$. We consider an equidistant distribution for comparison purposes. As mentioned above in Remark 6.1 1 the operator norm decreases in the equidistant setting when $\ell$ is increased, solving (6.2) by the least squares method. In numerical tests, it has turned out that choosing $\ell=1000$ equidistant $\beta_{j}$ for the unoptimized comparison operator gives among other choices of $\ell$ slightly better results with respect to the term (7.1). The left table in Table 1 shows the estimates of the operator norms of this unoptimized operator for $m=1,2,3$. The operator norms of the Hestenes extension stemming from the optimization process in Subsection 7.1 are listed in the right table in Table 1, The values and the distribution of the optimized $\beta_{j}, j=1, \ldots, \ell$, are provided in Figure 6 as well as the corresponding $\alpha_{j}, j=1, \ldots, \ell$, computed by (7.2).

Remark 7.2. We have not yet explicitly addressed the case $m=0$. Obviously, extending by zero yields an operator $Z: L^{2}(I) \rightarrow L^{2}(\tilde{I})$, with its adjoint given by the restriction $Z^{*}(g)=\left.g\right|_{I}$ for all $g \in L^{2}(\tilde{I})$. For $m=0$, one can also use the Hestenes operator for the case $m=1$ with $\beta_{2}$ chosen very small. Then the small support of $\eta_{\beta_{\ell}}^{\varepsilon}$ ensures that the extensions do not differ from the extensions by zero very much (see the central and right column in Figure 8), and additionally, continuous functions will have continuous extensions.

TABLE 1. Values for $F$ defined in (7.1), not optimized, equidistant distribution of the $\beta_{j}$ 's for $\ell=1000$ (left) and optimized for $\ell=2 \mathrm{~m}$ (right).

\begin{tabular}{|r|c|}
\hline$m$ & $\sqrt{\ell} \frac{\left(-\beta_{1}\right)^{m-\frac{1}{2}}}{\left(-\beta_{\ell}\right)^{m}}\|\alpha\|_{2}$ \\
\hline 1 & $5.72 e+2$ \\
2 & $1.70 e+5$ \\
3 & $5.33 e+7$ \\
\hline
\end{tabular}

\begin{tabular}{|r|c|}
\hline$m$ & $\sqrt{\ell} \frac{\left(-\beta_{1}\right)^{m-\frac{1}{2}}}{\left(-\beta_{\ell}\right)^{m}}\|\alpha\|_{2}$ \\
\hline 1 & $8.69 e+0$ \\
2 & $1.14 e+3$ \\
3 & $1.39 e+5$ \\
\hline
\end{tabular}




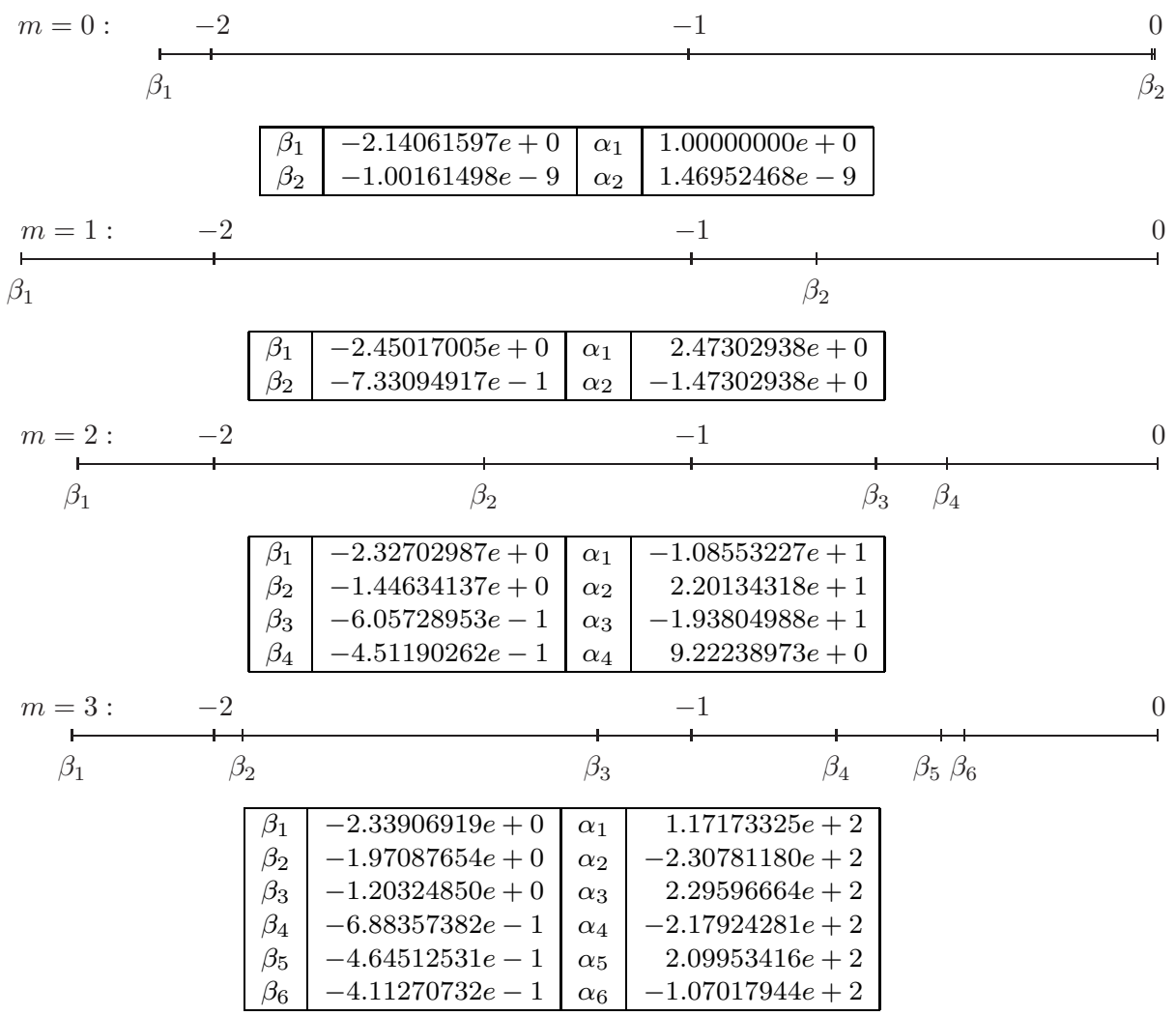

Figure 6. Optimized $\beta_{j}$, their distribution, and corresponding $\alpha_{j}$ solving (6.2) for $m=0, \ldots, 3$.
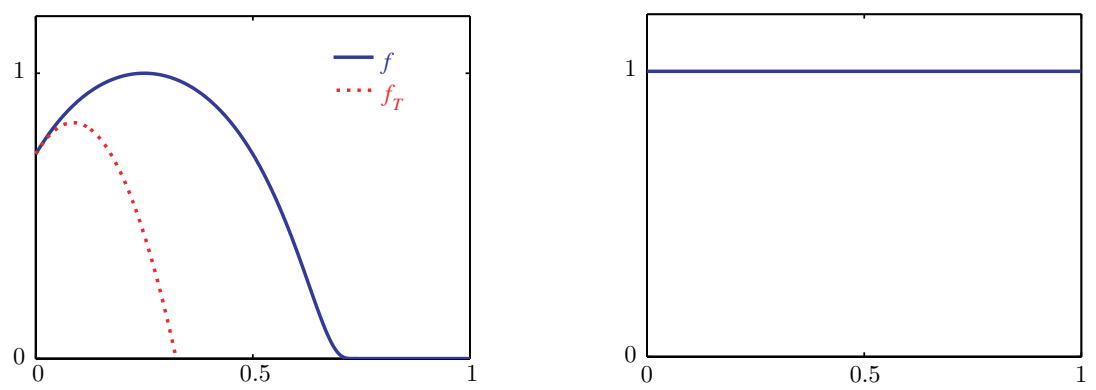

Figure 7. The function $f$ defined in (7.6), its Taylor expansion $f_{T}$ (left), and $g=\chi_{[0,1]}$ (right).

Numerical Examples. We briefly pause to illustrate the optimized extension and to consider extensions for two smooth functions and of the characteristic function. 

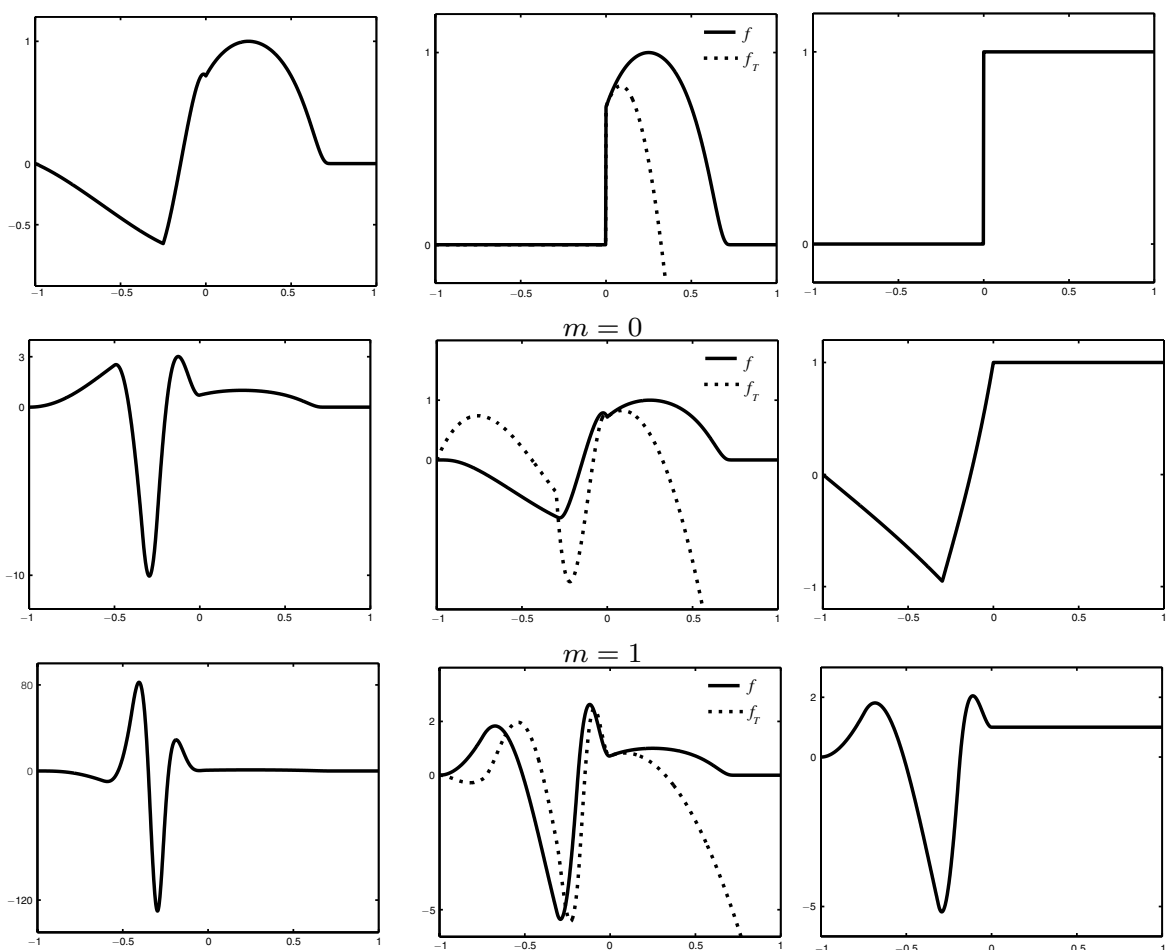

$m=1$
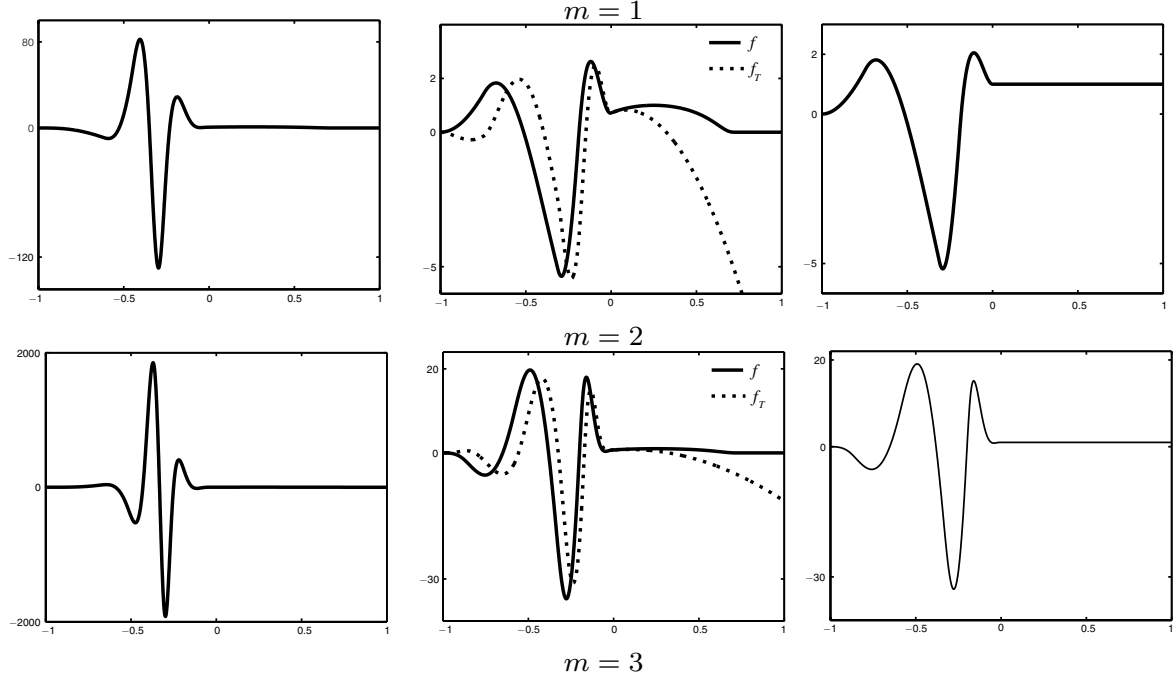

Figure 8. Left column: extensions of $f$ not optimized, equidistant distribution of the $\beta_{j}$ 's for $\ell=1000$; Center column: extensions of $f$ and $f_{T}$ optimized; Right column: extensions of $\chi_{[0,1]}$ optimized, $m=0, \ldots, 3$.

Define

$$
\begin{gathered}
f(x):= \begin{cases}\exp \left(1-\frac{1}{1-\left(2 x-\frac{1}{2}\right)^{2}}\right), & x \in\left(0, \frac{3}{4}\right), \\
0, & x \in\left[\frac{3}{4}, 1\right),\end{cases} \\
f_{T}(x):=e^{-\frac{1}{3}\left(1+\frac{32}{9} x-\frac{13 \cdot 128}{81} x^{2}\right),} \quad g:=\chi_{[0,1]} ;
\end{gathered}
$$

see Figure 7. $f_{T}$ is constructed to be the Taylor expansion of $f$ up to order 2 at $x=0$. Figure 8 shows extensions of $f, f_{T}$ and $g$. The center column displays extensions of $f$ and its Taylor expansion $f_{T}$ by the optimized Hestenes extension. In contrast to the trace dependent operators discussed in Section 4, the extensions 
of $f$ and $f_{T}$ differ in spite of coinciding trace values. For comparison, extensions of $f$ by the unoptimized Hestenes extension are displayed in the left column. The right column show extensions of $g=\chi_{[0,1]}$.

7.3. Optimization for $\ell>2 m$. As mentioned in Remark 6.1,1, smaller solutions of $\alpha$ with respect to the Euclidean norm can be obtained in the equidistant case by increasing $\ell$. This suggests applying a gradient method to the functional (7.1) for the general case $\ell>2 m$ to obtain further improvements. Although in this setting the representation (7.2) still solves (6.2), it does not coincide with the least squares solution $\alpha=V_{m}^{T}\left(V_{m} V_{m}^{T}\right)^{-1} b$ introduced in (6.6) (with $V_{m}$ from (6.3)); hence a minimization of the operator norm cannot be carried out analogously. We use an implicit gradient method instead, using the expression proved in [KS, Remark 10.1]. We apply this general formula to the situation of the least squares solution (6.6) in order to explicitly compute the gradient of (7.1) for the case $\ell>2 m=: \nu$. Defining $V:=V_{m}$ as in (6.3), we want to compute the gradient of the function

$$
\beta \mapsto \alpha(\beta):=V^{T}\left(V V^{T}\right)^{-1} b,
$$

where here

$$
A:=V V^{T}=\left(\sum_{q=1}^{\ell} \beta_{q}^{-\nu+i+j-2}\right)_{i j=1}^{\nu} .
$$

The proof of the following technical lemma can be found in [KS].

Lemma 7.3. The partial derivatives of the function defined in (7.7) are given by

$$
\partial_{\beta_{k}} \alpha_{j}= \begin{cases}\sum_{s=1}^{\nu}(\mu+s) \beta_{j}^{\mu+s-1} \sum_{t=1}^{\nu} a^{s t} b_{t} & \\ \quad+\sum_{s=1}^{\nu} \beta_{j}^{\mu+s} \sum_{t=1}^{\nu} b_{t} \sum_{q, r=1}^{\nu}-a^{s q} a^{r t}(2 \mu+q+r) \beta_{k}^{2 \mu+q+r-1}, & k=j \\ \sum_{s=1}^{\nu} \beta_{j}^{\mu+s} \sum_{t=1}^{\nu} s \sum_{q, r=1}^{\nu}-a^{s q} a^{r t}(2 \mu+q+r) \beta_{k}^{2 \mu+q+r-1}, & k \neq j .\end{cases}
$$

With these partial derivatives at hand we can compute the gradient of the functional (7.1) in order to perform a gradient descent as before for the case $\ell=2 \mathrm{~m}$. Various results of this optimization procedure for different choices of $\ell$ show that this relaxation yields larger operator norms of the Hestenes extension; see Table 2 . Now after we have constructed an optimized extension operator satisfying (A), we can proceed with the construction of wavelets.

TABLE 2. Optimized operator norms $\sqrt{\ell} \frac{\left(-\beta_{1}\right)^{m-\frac{1}{2}}}{\left(-\beta_{\ell}\right)^{m}}\|\alpha\|_{2}$ for $m=$ $1,2,3, \ell \geq 2 m$.

\begin{tabular}{|c|c|c|c|c|c|c|c|}
\hline$m \backslash \ell$ & 2 & 3 & 4 & 5 & 6 & 7 & 8 \\
\hline 1 & 8.69 & 8.97 & $9.29 e+0$ & $1.00 e+1$ & $1.05 e+1$ & $9.82 e+1$ & $1.03 e+1$ \\
2 & & & $1.14 e+3$ & $1.36 e+3$ & $1.51 e+4$ & $1.78 e+3$ & $2.01 e+3$ \\
3 & & & & & $1.39 e+5$ & $1.47 e+5$ & $1.68 e+5$ \\
\hline
\end{tabular}




\section{Construction of the Wavelets}

In this section we summarize the construction of wavelets on manifolds in [DS]. We start by subdividing the manifold into quadrangular patches. In a second step, wavelets will be pushed forward to these patches from the parameter domain in Subsection 8.4. Finally these wavelets are extended appropriately to the manifold in a third stage in Subsection 8.5.

8.1. Deconstruction of the manifold. We consider manifolds $\Gamma$ that are the disjoint union of parametric images of the open unit cube $\square=(0,1)^{n}$ where $n$ is the dimension of the manifold; i.e., we assume that we have sufficiently smooth mappings $\kappa_{i}: \square \rightarrow \Gamma, \Gamma_{i}:=\kappa_{i}(\square)$ and

$$
\Gamma=\bigcup_{i=1}^{N} \overline{\Gamma_{i}}, \Gamma_{i} \cap \Gamma_{j}=\emptyset \quad \text { for } i \neq j .
$$

Remark 8.1. For technical reasons we require the following property. If $x \in \Gamma$ is a vertex of some patch $\Gamma_{i}$, then it is also a vertex of any other patch $\Gamma_{j}$ with $x \in \overline{\Gamma_{j}}$.

$\Gamma$ has a boundary; we denote the part where Dirichlet boundary conditions shall be applied by $\partial_{D}$. Likewise, $\partial_{N}$ denotes the part of the boundary of $\Gamma$ where Neumann boundary conditions will be posed.

Remark 8.2. The definition of parametric mappings imply that for all $i=1, \ldots, N$ we have a square $\diamond \ni \square$ and a bijective mapping $\hat{\kappa}_{i}$ with the same smoothness as the $\kappa_{i}$ such that $\left.\hat{\kappa}_{i}\right|_{\square}=\kappa_{i}$. $\square \Subset \diamond$ means that $\square$ is compactly contained in $\diamond$ and hence there exist $\varepsilon_{i}^{a}, \varepsilon_{i}^{b}>0$ such that $\square \subset\left(-\varepsilon_{i}^{a}, 1+\varepsilon_{i}^{b}\right)^{n} \subset \diamond$. Hence we can assume without loss of generality that

$$
\diamond=\times_{i=1}^{n}\left(-\varepsilon_{i}^{a}, 1+\varepsilon_{i}^{b}\right)=: \square_{N}^{(i)}
$$

This is more specific than in [DS] and will play an important role later.

The manifold $\Gamma$ is equipped with the scalar product

$$
\langle u, v\rangle_{\Gamma}=\int_{\Gamma} u v d \mu
$$

derived by integrals over the patches $\Gamma_{i}$,

$$
\int_{\Gamma_{i}} u d \mu:=\int_{\square} u \kappa_{i} \sqrt{\left|\operatorname{det} \nabla \kappa_{i}^{T} \nabla \kappa_{i}\right|} d L^{n} .
$$

We abbreviate

$$
\partial \kappa_{i}:=\sqrt{\left|\operatorname{det} \nabla \kappa_{i}^{T} \nabla \kappa_{i}\right|}
$$

Recall the special numbering of the patches given in $\mathrm{DS}$. The numbering has two phases, an initialization phase and an induction phase. In the initialization phase we pick an arbitrary patch and call it $\Gamma_{1}$. We set $\mathcal{G}_{1}:=\left\{\Gamma_{1}\right\}$. In the induction phase, let $\mathcal{G}_{i}$ be constructed. We then set

$$
\mathcal{G}_{i+1}:=\mathcal{G}_{i} \cup\left\{\text { all patches that share an edge with patches in } \mathcal{G}_{i}\right\}
$$


and number the new patches in $\mathcal{G}_{i+1}$ (i.e. the patches in $\mathcal{G}_{i+1} \backslash \mathcal{G}_{i}$ ) as follows: take any patch that has not been numbered yet and assign it the next free number. Repeat this process until all patches in $\mathcal{G}_{i+1}$ are numbered. In the rest of the paper the numbering $\left(\Gamma_{i}\right)_{i=1}^{N}$ will always be assumed to stem from this ordering. An example can be seen in Figure 12 below.

8.2. Neighbours, inflow and outflow. To any patch $\Gamma_{i}$ we associate a set of inflow and outflow neighbours. We divide the boundary of $\Gamma_{i}$ into an inflow and an outflow boundary. The outflow boundary $\partial^{\uparrow} \Gamma_{i}$ is defined by

$$
\partial^{\uparrow} \Gamma_{i}:=\bigcup\left\{\text { edges } \nu \text { of } \Gamma_{i}:\left(\nu \text { is also an edge of } \Gamma_{j} \text { with } j>i\right) \text { or } \nu \subset \partial_{N}\right\} .
$$

Analogously we define the inflow boundary $\partial^{\downarrow} \Gamma_{i}$ to be

(8.4) $\partial^{\downarrow} \Gamma_{i}:=\bigcup\left\{\right.$ edges $\nu$ of $\Gamma_{i}:\left(\nu\right.$ is also an edge of $\Gamma_{j}$ with $\left.j<i\right)$ or $\left.\nu \subset \partial_{D}\right\}$.

We will later define primal wavelets on $\Gamma$ which have zero boundary values on the inflow boundary of $\Gamma_{i}$ on the primal side. For this reason edges on the Dirichlet boundary are assigned to the inflow boundary. Outflow neighbours $\mathcal{N}_{i}^{\uparrow}$ and inflow neighbours $\mathcal{N}_{i}^{\downarrow}$ are now defined by

$$
\mathcal{N}_{i}^{\uparrow}:=\left\{\Gamma_{j} \subset \Gamma: j>i, \overline{\Gamma_{j}} \cap\left(\text { rel int } \partial^{\uparrow} \Gamma_{i}\right) \neq \emptyset\right\}
$$

and

$$
\mathcal{N}_{i}^{\downarrow}:=\left\{\Gamma_{j} \subset \Gamma: j<i, \overline{\Gamma_{j}} \cap\left(\text { rel int } \partial^{\downarrow} \Gamma_{i}\right) \neq \emptyset\right\} .
$$

The outflow domain $\Gamma_{i}^{\uparrow}$ and the inflow domain $\Gamma_{i}^{\downarrow}$ of $\Gamma_{i}$ will be denoted by

$$
\Gamma_{i}^{\uparrow}:=\operatorname{int}\left(\overline{\Gamma_{i}} \cup\left\{\overline{\Gamma^{\prime}}: \Gamma^{\prime} \in \mathcal{N}_{i}^{\uparrow}\right\}\right), \quad \Gamma_{i}^{\downarrow}:=\operatorname{int}\left(\overline{\Gamma_{i}} \cup\left\{\overline{\Gamma^{\prime}}: \Gamma^{\prime} \in \mathcal{N}_{i}^{\downarrow}\right\}\right),
$$

respectively. Then the neighbourhood domain $\Gamma_{i}^{N}$ of $\Gamma_{i}$ is

$$
\Gamma_{i}^{N}:=\operatorname{int} \overline{\Gamma_{i}^{\uparrow}} \cup \overline{\Gamma_{i}^{\downarrow}}
$$

As we want to push wavelets on $\square$ satisfying certain boundary conditions forward to the patches $\Gamma_{i}$, we have to pull back the boundary conditions of $\Gamma_{i}$ to $\square$. This can be done as follows. To each patch $\Gamma_{i}$ assign a set

$$
\mathbf{Z}^{(i)}=\left(Z_{1}^{(i)}, \ldots, Z_{N}^{(i)}\right) \subset\{0,1\}^{n},
$$

where the $Z_{j}^{(i)} \subset\{0,1\}$ are characterized by

$$
\partial_{\mathbf{Z}^{(i)}} \square:=\kappa_{i}^{-1}\left(\partial^{\downarrow} \Gamma_{i}\right)=\bigcup_{j=1}^{n}[0,1]^{j-1} \times Z_{j}^{(i)} \times[0,1]^{n-j} ;
$$

i.e., the $\mathbf{Z}^{(i)}$ encode the preimage of the inflow boundary $\partial^{\downarrow} \Gamma_{i}$. Analogously, $\tilde{\mathbf{Z}}^{(i)}:=$ $\left(\{0,1\} \backslash Z_{1}^{(i)}, \ldots,\{0,1\} \backslash Z_{N}^{(i)}\right)$ encodes the preimage of the outflow boundary $\partial^{\uparrow} \Gamma_{i}$, denoted by $\partial_{\tilde{\mathbf{Z}}^{(i)}} \square$. With the $\varepsilon_{i}^{a}, \varepsilon_{i}^{b}$ from Remark 8.2 and

$$
[0,1]_{Z_{i}}:=\left\{\begin{array}{ll}
{[0,1],} & \text { if } Z=\emptyset, \\
{\left[-\varepsilon_{i}^{a}, 1\right],} & \text { if } Z=\{0\}, \\
{\left[0,1+\varepsilon_{i}^{b}\right],} & \text { if } Z=\{1\}, \\
{\left[-\varepsilon_{i}^{a}, 1+\varepsilon_{i}^{b}\right],} & \text { if } Z=\{0,1\},
\end{array} \quad \text { for } Z \subset\{0,1\},\right.
$$


we define, in analogy to $\Gamma_{i}^{\downarrow}$,

$$
\square_{\mathbf{Z}^{(i)}}:=[0,1]_{Z_{1}^{(i)}} \times \cdots \times[0,1]_{Z_{N}^{(i)}}
$$

and $\square_{\tilde{\mathbf{Z}}^{(i)}}$ analogously plays the part of $\Gamma_{i}^{\uparrow}$ in the parameter domain. Obviously, the analogue to $\Gamma_{i}^{N}$ from (8.8) is $\square_{i}^{N}=\left[-\varepsilon_{1}^{a}, 1+\varepsilon_{1}^{b}\right] \times \cdots \times\left[-\varepsilon_{n}^{a}, 1+\varepsilon_{n}^{b}\right]$ defined in (8.1). This notion is needed to define wavelets on $\square$ with certain complementary boundary conditions.

8.3. Wavelets on $\square$. In [DS2], biorthogonal wavelet bases of $\square$ with certain complementary boundary conditions were introduced for use in [DS]. Here we only focus on the most important properties of these wavelet bases. To characterize the complementary boundary conditions mentioned above, we introduce for $\mathbf{Z}$ as in (8.9) and $\square_{\mathbf{Z}}$ from (8.12) the Sobolev spaces with built-in boundary values

$$
H^{m}(\square)_{\mathbf{z}}:=\left\{f \in H^{m}(\square): \chi_{\square} f \in H^{m}\left(\square_{\mathbf{z}}\right)\right\} .
$$

Here $\chi_{\square} f$ denotes the extension of $f$ by zero to $\square_{\mathbf{z}}$.

Remark 8.3. (1) The spaces $H^{m}(\square)_{\mathbf{Z}^{(i)}}$ consist of functions which vanish in the $H^{m}$-sense on the retract of the inflow boundary of $\Gamma_{i}$. On the retract of the outflow boundary we have free boundary conditions.

(2) The spaces $H^{m}(\square)_{\tilde{\mathbf{Z}}^{(i)}}$ consist of functions which vanish in $H^{m}$-sense on the retract of the outflow boundary of $\Gamma_{i}$. On the retract of the inflow boundary we have free boundary conditions. In this sense, $H^{m}(\square) \mathbf{Z}^{(i)}$ and $H^{m}(\square)_{\tilde{\mathbf{Z}}^{(i)}}$ contain complementary boundary conditions.

(3) In $\left[\overline{D S 2}\right.$, biorthogonal wavelet bases $\Psi^{\mathbf{Z}}$ of $H^{m}(\square)_{\mathbf{Z}^{(i)}}, \tilde{\Psi}^{\tilde{\mathbf{z}}}$ of $H^{m}(\square)_{\tilde{\mathbf{Z}}}(i)$ were constructed for arbitrary $\mathbf{Z}$. This means we have for $i=1, \ldots, N$ wavelet bases $\Psi^{\mathbf{Z}^{(i)}}, \tilde{\Psi}^{\mathbf{Z}^{(i)}}$. $\Psi^{\mathbf{Z}^{(i)}}$ has zero boundary values on the inflow and $\tilde{\Psi}^{\tilde{\mathbf{Z}}^{(i)}}$ has zero boundary values on the outflow, and $\Psi^{\mathbf{Z}^{(i)}}$ and $\tilde{\Psi}^{\tilde{\mathbf{z}}^{(i)}}$ are biorthogonal. More properties of $\Psi^{\mathbf{Z}^{(i)}}, \tilde{\Psi}^{\tilde{\mathbf{Z}}^{(i)}}$ are found in [DS2].

8.4. Wavelets on $\Gamma_{i}$. The wavelets $\Psi^{\mathbf{Z}^{(i)}}, \tilde{\Psi}^{\mathbf{\mathbf { Z }}^{(i)}}$ on $\square$ introduced in Subsection 8.3 shall be pushed forward to $\Gamma_{i}$ using the parametric mapping $\kappa_{i}$. The boundary conditions in the flat case (8.13) have the following analogue on the manifold:

$$
\begin{aligned}
& H^{m}\left(\Gamma_{i}\right)^{\uparrow}:=\left\{f \in H^{m}\left(\Gamma_{i}\right): \chi_{\Gamma_{i}} f \in H^{m}\left(\Gamma_{i}^{\uparrow}\right)\right\}, \\
& H^{m}\left(\Gamma_{i}\right)^{\downarrow}:=\left\{f \in H^{m}\left(\Gamma_{i}\right): \chi_{\Gamma_{i}} f \in H^{m}\left(\Gamma_{i}^{\downarrow}\right)\right\} .
\end{aligned}
$$

We define wavelets on the patches $\Gamma_{i}$ by

$$
\Psi^{\Gamma_{i} \downarrow}:=\Psi^{\mathbf{Z}^{(i)}} \circ \kappa_{i}^{-1}, \quad \tilde{\Psi}^{\Gamma_{i} \uparrow}:=\tilde{\Psi}^{\tilde{\mathbf{Z}}^{(i)}} \circ \kappa_{i}^{-1} .
$$

Remark 8.4. By Remark 8.3, it is clear that

$$
\Psi^{\Gamma_{i} \downarrow} \subset H^{m}\left(\Gamma_{i}\right)^{\downarrow}, \quad \tilde{\Psi}^{\Gamma_{i} \uparrow} \subset H^{m}\left(\Gamma_{i}\right)^{\uparrow},
$$

but not necessarily

$$
\Psi^{\Gamma_{i} \downarrow} \subset H^{m}(\Gamma), \quad \tilde{\Psi}^{\Gamma_{i} \uparrow} \subset H^{m}(\Gamma) .
$$


In fact, when $\Gamma_{i}$ has a nonempty outflow boundary, by Remark 8.311 the primal wavelets $\Psi^{\Gamma_{i} \downarrow}$ have free boundary conditions there. Due to the basis property Remark 8.313 there must be wavelets $\psi \in \Psi^{\Gamma_{i} \downarrow}$ that do not vanish on $\partial^{\uparrow} \Gamma_{i}$. But then the extension $\chi_{\Gamma_{i}} \psi$ by zero onto the whole of $\Gamma$ is not continuous in $\Gamma$, hence $\chi_{\Gamma_{i}} \psi \notin H^{m}(\Gamma)$ for $m \geq 1$. Complementary observations hold for the dual wavelets $\tilde{\Psi}^{\Gamma_{i} \uparrow}$

Remark 8.5. Due to Remark 8.4, we must extend the wavelets $\Psi^{\Gamma_{i} \downarrow}$ over the outflow boundary of $\Gamma_{i}$, and the wavelets $\tilde{\Psi}^{\Gamma_{i} \uparrow}$ over the inflow boundary to the whole of $\Gamma$ in some appropriate way. So we need extension operators

$$
E_{i}: H^{m}\left(\Gamma_{i}\right) \rightarrow H^{m}\left(\Gamma_{i}^{\uparrow}\right), \quad \tilde{E}_{i}: H^{m}\left(\Gamma_{i}\right) \rightarrow H^{m}\left(\Gamma_{i}^{\downarrow}\right) .
$$

To achieve $E \psi \in H^{m}(\Gamma)$ for all $\psi \in \Psi^{\Gamma_{i} \downarrow}$, we additionally have the requirement

$$
E_{i} f \text { has zero boundary values on } \partial \Gamma_{i}^{\uparrow} \backslash \partial \Gamma_{i} .
$$

Then for $\psi \in \Psi^{\Gamma_{i} \downarrow}$, we have $E_{i} \psi \in H^{m}(\Gamma)$ as $\psi$ already has zero boundary values on the inflow boundary.

8.5. Wavelets on $\Gamma$. Next we extend the wavelets $\Psi^{\Gamma_{i} \downarrow}, \tilde{\Psi}^{\Gamma_{i} \uparrow}$ defined in 8.15) to the whole of $\Gamma$. We will derive extension operators $E_{i}: H^{m}\left(\Gamma_{i}\right) \rightarrow H^{m}\left(\Gamma_{i}^{\uparrow}\right)$ satisfying the analogous property to the univariate Property $(\mathrm{A})$ for the multivariate case, called property $(\mathrm{E})$ :

(L) Locality: $E_{i}$ satisfies (8.18).

(CE) Continuity: $E_{i}: H^{m}\left(\Gamma_{i}\right) \rightarrow H^{m}\left(\Gamma_{i}^{\uparrow}\right)$ is continuous.

$\left(\mathrm{CE}^{*}\right)$ Continuity of $E_{i}^{*}: E_{i}^{*}: H^{m}\left(\Gamma_{i}^{\uparrow}\right) \rightarrow H^{m}\left(\Gamma_{i}\right)$ satisfies

$$
\left\|E_{i}^{*} f\right\|_{H^{m}\left(\Gamma_{i}\right)^{\uparrow}} \lesssim\|f\|_{H^{m}\left(\Gamma_{i}^{\uparrow}\right)} \quad \text { for all } f \in H^{m}\left(\Gamma_{i}^{\uparrow}\right) .
$$

$\left(\mathrm{CE}^{*}\right)$ means that $E_{i}^{*}$ continuously pulls back functions living on the outflow of $\Gamma_{i}$ back to $\Gamma_{i}$ such that the pullback has zero boundary values on the outflow boundary.

We will define the $E_{i}$ as tensor products of the Hestenes extension $A$ defined in Section 6. Note that we could take any extension operator $A$ satisfying (A). The constructive proof of the following lemma is given in [KS].

Lemma 8.6. For any $\mathbf{Z}$ as in (8.12) we have an extension operator

$$
A_{\mathbf{Z}}: H^{m}(\square) \rightarrow H^{m}\left(\square_{\mathbf{Z}}\right)
$$

such that $A_{\mathbf{Z}} f$ has zero boundary values on $\partial \square_{\mathbf{Z}} \backslash \partial \square$.

Now extensions of $f \in H^{m}\left(\Gamma_{i}\right)$ to $H^{m}\left(\Gamma_{i}^{\uparrow}\right)$ can be defined by first pulling back functions $f \in H^{m}\left(\Gamma_{i}\right)$ to the parameter domain $\square$, applying the Hestenes extension $A_{\tilde{\mathbf{Z}}^{(i)}}$ to the pullback and pushing forward the extension again. Define the pullback mapping $\kappa_{i}^{*}: H^{m}\left(\Gamma_{i}\right) \rightarrow H^{m}(\square)$ to be $\left(\kappa_{i}^{*} v\right)(x):=v\left(\kappa_{i}(x)\right)$. By Lemma 8.6 and the definition of $\mathbf{Z}$ in (8.12) we have $\operatorname{supp} A_{\tilde{\mathbf{Z}}^{(i)}} f \subset \square_{\mathbf{Z}^{(i)}} \subset \square_{N}^{(i)} \subset \diamond$ and, hence, we 
can push $A_{\tilde{\mathbf{Z}}^{(i)}} f$ forward to the manifold by applying $\left(\kappa_{i}^{\uparrow}\right)^{*}$. Here $\kappa_{i}^{\uparrow}:=\left.\hat{\kappa}_{i}\right|_{\tilde{\mathbf{Z}}^{(i)}}$ is the part of $\hat{\kappa}_{i}$ defined in Remark 8.2 that maps to the outflow domain $\Gamma_{i}^{\uparrow}$. For each $i=1, \ldots, N$ we define the Hestenes extension $E_{i}: H^{m}\left(\Gamma_{i}\right) \rightarrow H^{m}\left(\Gamma_{i}^{\uparrow}\right)$ by

$$
E_{i}:=\left(\kappa_{i}^{\uparrow}\right)^{*} \circ A_{\tilde{\mathbf{Z}}^{(i)}} \circ \kappa_{i}^{*}
$$

Theorem 8.7. $E_{i}$ fulfills the requirements $(\mathrm{L})$ and $(\mathrm{CE})$ by Lemma 6.3, and we even have $E_{i} \psi \in H^{m}(\Gamma)$ for $\psi \in \Psi^{\Gamma_{i} \downarrow}$ by Remark 8.5.

The key operators $P_{i}: L^{2}(\Gamma) \rightarrow L^{2}(\Gamma)$, which serve to extend the wavelets $\Psi^{\Gamma_{i} \downarrow}$ onto the whole of $\Gamma$, are defined inductively by $P_{1} v:=\chi_{\Gamma_{i}^{\uparrow}} E_{1}\left(\left.v\right|_{\Gamma_{i}}\right)$, and

$$
P_{i} v:=\chi_{\Gamma_{i}}^{\uparrow} E_{i}\left(\left.\left(v-\sum_{j<i} P_{j} v\right)\right|_{\Gamma_{i}}\right) \quad \text { for } i=2, \ldots, N .
$$

In [DS] the following results are proved.

Theorem 8.8. The adjoint $P^{*}$ of $P$ relative to the $L^{2}$ inner product (8.2) is given by

$$
P_{i}^{*} w=\left(I-\sum_{j<i} P_{j}^{*}\right) \chi_{\Gamma_{i}} E_{i}^{*}\left(\left.w\right|_{\Gamma_{i}^{\uparrow}}\right)
$$

where by (8.20) and (8.2) $E_{i}^{*}$ is given by $E_{i}^{*}=\left(\kappa_{i}^{-1}\right)^{*}\left(\partial \kappa^{-1} A_{\tilde{\mathbf{Z}}^{(i)}}^{*} \partial \kappa_{i}^{\uparrow}\left(\kappa_{i}^{\uparrow}\right)^{*}\right)$. Due to Lemma 6.3. $E_{i}^{*}$ satisfies $\left(\mathrm{CE}^{*}\right)$ and, hence, $(\mathrm{E})$ is satisfied. For $f \in H^{m}\left(\Gamma_{i}\right)^{\downarrow}$ we have $P_{i} f=E_{i} f \in H^{m}(\Gamma)$, and for $f \in H^{m}\left(\Gamma_{i}\right)^{\uparrow}$ we have $P_{i}^{*} f \in H^{m}(\Gamma)$. Hence the operator $P_{i}^{*}$ takes on the role of $\tilde{E}_{i}$.

Consequently, defining wavelets $\Psi_{i}^{\Gamma} \subset H^{m}(\Gamma), \tilde{\Psi}_{i}^{\Gamma} \subset H^{m}(\Gamma)$ on $\Gamma$ by

$$
\Psi_{i}^{\Gamma}:=P_{i} \chi_{\Gamma_{i}} \Psi^{\Gamma_{i} \downarrow}, \quad \tilde{\Psi}_{i}^{\Gamma}:=P_{i}^{*} \chi_{\Gamma_{i}} \tilde{\Psi}^{\Gamma_{i} \uparrow}
$$

with the $\Psi^{\Gamma_{i} \downarrow}$ and $\tilde{\Psi}^{\Gamma_{i} \uparrow}$ from 8.15 is justified. With $E_{i}$ satisfying (E) due to Theorem 8.7 and Theorem 8.8 it is possible following the proof in DS] to obtain the following result, where further properties such as approximation inequalities and polynomial exactness of these bases can also be found.

Theorem 8.9. The wavelets $\Psi^{\Gamma}:=\Psi_{1}^{\Gamma} \cup \cdots \cup \Psi_{N}^{\Gamma}, \tilde{\Psi}^{\Gamma}:=\tilde{\Psi}_{1}^{\Gamma} \cup \cdots \cup \tilde{\Psi}_{N}^{\Gamma}$ constitute biorthogonal wavelet bases of $H^{m}(\Gamma)$ (i.e., $\left.\left\langle\Psi^{\Gamma}, \tilde{\Psi}^{\Gamma}\right\rangle_{\Gamma}=\mathbf{I}\right)$, realizing norm equivalences $\|v\|_{H^{m}(\Gamma)}^{2} \sim \sum_{j=j_{0}}^{\infty} 2^{2 j m}\left\|\left\langle v,\left(\Psi^{\Gamma}\right)_{j}\right\rangle_{L^{2}(\Gamma)}\right\|_{\ell_{2}}^{2}$ for all $v \in H^{m}(\Gamma)$, where $\left(\Psi^{\Gamma}\right)_{j}$ are all wavelets of level $j$ from the set $\Psi^{\Gamma}$.

\section{Biorthogonal WAVELET BASES FOR THE SPHERE}

Finally we construct and plot biorthogonal wavelet bases for the sphere $S^{2}:=$ $\left\{x \in \mathbb{R}^{3}:\|x\|_{2}=1\right\}$. First we introduce a suitable quadrangulation of the sphere using six quadrangles which satisfies the property required by Remark 8.1. 


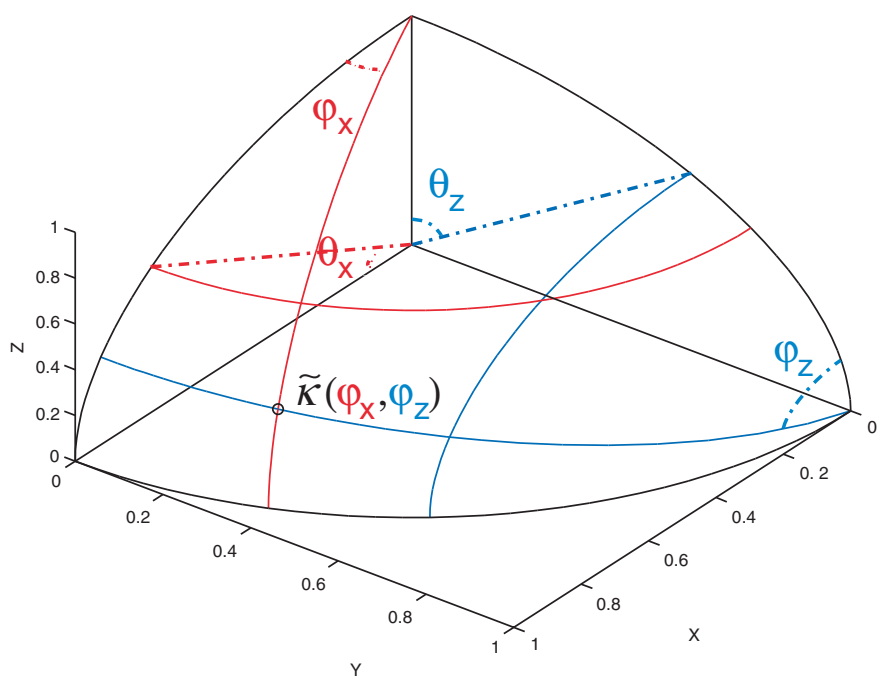

FiguRE 9 . The polar coordinates $\left(\varphi_{x}, \theta_{x}\right)$ around the $z$-pole and $\left(\varphi_{z}, \theta_{z}\right)$ around the $y$-pole.

9.1. A quadrangulation of the sphere. One possibility for defining a quadrangulation of the sphere is to introduce the polar coordinates $\left(\varphi_{x}, \theta_{x}\right)$ around the $z$-pole and $\left(\varphi_{z}, \theta_{z}\right)$ around the $y$-pole; see Figure 9. We see that $\varphi_{x}$ describes a positive angle towards the $z x$-plane and $\varphi_{z}$ describes a positive angle towards the $y z$-plane. Hence these angles describe great circles $G_{x}\left(\varphi_{x}\right), G_{z}\left(\varphi_{z}\right)$, respectively, which lie in the planes $E_{x}, E_{z}$ with normal vectors $\nu_{x}, \nu_{z}$. We easily see that

$$
\begin{aligned}
& \nu_{x}=\left(\begin{array}{ccc}
\cos \varphi_{x} & -\sin \varphi_{x} & 0 \\
\sin \varphi_{x} & \cos \varphi_{x} & 0 \\
0 & 0 & 1
\end{array}\right), \quad e_{2}=\left(\begin{array}{c}
-\sin \varphi_{x} \\
\cos \varphi_{x} \\
0
\end{array}\right) \text {, } \\
& \nu_{z}=\left(\begin{array}{ccc}
\cos \varphi_{z} & 0 & \sin \varphi_{z} \\
0 & 1 & 0 \\
-\sin \varphi_{z} & 0 & \cos \varphi_{z}
\end{array}\right), \quad e_{1}=\left(\begin{array}{c}
\cos \varphi_{z} \\
0 \\
-\sin \varphi_{z}
\end{array}\right) \text {, }
\end{aligned}
$$

i.e., $\nu_{x}$ is the unit vector $e_{2}$ rotated by the angle $\varphi_{x}$ around the $z$-axis and $\nu_{z}$ is the unit vector $e_{1}$ rotated by the angle $\varphi_{z}$ around the $x$-axis. Any point $(x, y, z) \in$ $S^{2} \backslash\{(0,0,1),(0,1,0)\}$ can be expressed uniquely by means of the polar coordinates $\left(\varphi_{x}, \theta_{x}\right),\left(\varphi_{z}, \theta_{z}\right)$; i.e.,

$$
\left(\begin{array}{c}
x \\
y \\
z
\end{array}\right)=\left(\begin{array}{c}
\cos \varphi_{x} \cos \theta_{x} \\
\sin \varphi_{x} \cos \theta_{x} \\
\sin \theta_{x}
\end{array}\right)=\left(\begin{array}{c}
\sin \varphi_{z} \cos \theta_{z} \\
\sin \theta_{z} \\
\cos \varphi_{z} \cos \theta_{z}
\end{array}\right) .
$$

The idea of the quadrangulation introduced next is the following. We aim at finding six quadrangular patches, each centered at the positive and negative poles of each dimension, i.e., at $(1,0,0),(-1,0,0),(0,1,0),(0,-1,0),(0,0,1),(0,0,-1)$. We concentrate on the patch centered at the $x$-pole $(1,0,0)$, called $\Gamma_{1}$ later. All other patches are just rotations of this patch. $\varphi_{x}$ and $\varphi_{z}$ will play the role of coordinates 


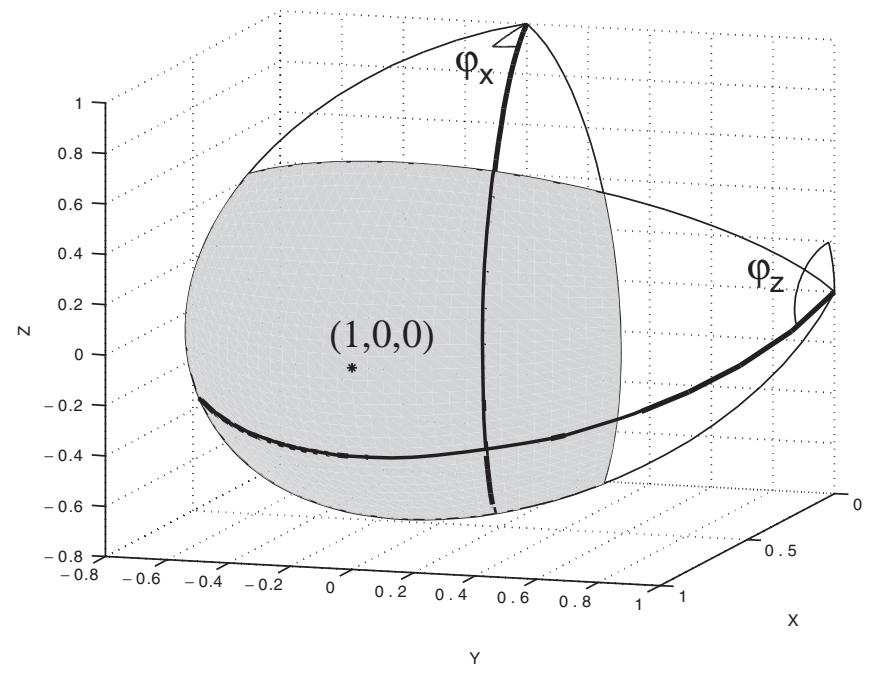

Figure 10. Coordinates $\tilde{\kappa}\left(\varphi_{x}, \varphi_{z}\right)$ forming Patch 1 centered at $(1,0,0)$.

in the parametrization $\tilde{\kappa}$, which is defined by

$$
\tilde{\kappa}\left(\theta_{x}, \theta_{z}\right):=\left\{\begin{array}{l}
\text { the point } \tilde{x} \text { with } \tilde{x}_{1}>0 \text { where the great circles } \\
G_{x}\left(\varphi_{x}\right), G_{z}\left(\varphi_{z}\right), \text { described by } \varphi_{x}, \varphi_{z}, \text { meet. }
\end{array}\right\}
$$

The intersection point $\tilde{\kappa}\left(\varphi_{x}, \varphi_{z}\right)$ is displayed in Figure 9 ,

Lemma 9.1. We have

$$
\tilde{\kappa}\left(\varphi_{x}, \varphi_{z}\right)=\frac{1}{\sqrt{\sin ^{2} \varphi_{z}+\cos ^{2} \varphi_{x} \cos ^{2} \varphi_{z}}}\left(\begin{array}{c}
\cos \varphi_{x} \sin \varphi_{z} \\
\sin \varphi_{x} \sin \varphi_{z} \\
\cos \varphi_{x} \cos \varphi_{z}
\end{array}\right) .
$$

Proof. The points where the great circles $G_{x}\left(\varphi_{x}\right)$ and $G_{z}\left(\varphi_{z}\right)$ meet are the same as the points where the intersection line of the planes $E_{x}$ and $E_{z}$ meet the sphere. The intersection line of the planes is given by the scalar multiples of the outer product $\nu_{x} \times \nu_{z}$ of their normals. Normalizing this outer product yields the point were the line hits the sphere and hence

$$
\tilde{\kappa}\left(\varphi_{x}, \varphi_{z}\right)=\frac{\nu_{x} \times \nu_{z}}{\left|\nu_{x} \times \nu_{z}\right|}=\frac{1}{\left|\nu_{x} \times \nu_{z}\right|}\left(\begin{array}{c}
\cos \varphi_{x} \sin \varphi_{z} \\
\sin \varphi_{x} \sin \varphi_{z} \\
\cos \varphi_{x} \cos \varphi_{z}
\end{array}\right),
$$

which proves the claim after calculating the norm of the vector $\nu_{x} \times \nu_{z}$.

Now we are ready to define the first patch $\Gamma_{1}$ as $\Gamma_{1}:=\tilde{\kappa}\left(\left(-\frac{\pi}{4}, \frac{\pi}{4}\right) \times\left(\frac{\pi}{4}, \frac{3}{2} \pi\right)\right)$. In Figure 10 we see $\Gamma_{1}$ with coordinates $\left(\varphi_{x}, \varphi_{z}\right) . \Gamma_{1}$ is centered at $(1,0,0)$. By the nature of the polar coordinates it is clear that $\Gamma_{1}$ is exactly a sixth of the sphere. As all parametric mappings shall map from $(0,1)^{2}$ we have to compose $\tilde{\kappa}$ with the linear reparametrization $\phi:(0,1)^{2} \rightarrow\left(-\frac{\pi}{4}, \frac{\pi}{4}\right) \times\left(\frac{\pi}{4}, \frac{3}{2} \pi\right)$ given by

$$
\phi(x, y)=\left(\frac{\pi}{2} x-\frac{\pi}{4}, \frac{\pi}{2} y+\frac{\pi}{4}\right)
$$

and define $\kappa_{1}:(0,1)^{2} \rightarrow \Gamma_{1}$ by $\kappa_{1}:=\tilde{\kappa} \circ \phi$. So far it is not clear that $\kappa_{1}$ is invertible. 
Lemma 9.2. The inverse of $\tilde{\kappa}$ is given by

$$
\tilde{\kappa}^{-1}\left(\begin{array}{l}
x \\
y \\
z
\end{array}\right)=\left(\begin{array}{l}
\arcsin \left(\frac{y}{\cos (\arcsin (z))}\right) \\
\arcsin \left(\frac{x}{\cos (\arcsin (y))}\right)
\end{array}\right)
$$

for $z \geq 0$ and

$$
\tilde{\kappa}^{-1}\left(\begin{array}{l}
x \\
y \\
z
\end{array}\right)=\left(\begin{array}{c}
\arcsin \left(\frac{y}{\cos (\arcsin (z))}\right) \\
\pi-\arcsin \left(\frac{x}{\cos (\arcsin (y))}\right)
\end{array}\right)
$$

for $z<0$.

Proof. According to (9.2), the point $(x, y, z)$ has the representation $\left(\varphi_{x}, \theta_{x}\right),\left(\varphi_{z}, \theta_{z}\right)$ in polar coordinates. The inverses of the polar coordinates for $z \geq 0$ are obviously given by

$$
\left(\begin{array}{c}
\varphi_{x} \\
\theta_{x}
\end{array}\right)=\left(\begin{array}{c}
\arcsin \left(\frac{y}{\cos \theta_{x}}\right) \\
\arcsin (z)
\end{array}\right), \quad\left(\begin{array}{c}
\varphi_{z} \\
\theta_{z}
\end{array}\right)=\left(\begin{array}{c}
\arcsin \left(\frac{x}{\cos \theta_{z}}\right) \\
\arcsin (y)
\end{array}\right),
$$

respectively. Inserting $\arcsin (z)$ for $\theta_{x}, \arcsin (y)$ for $\theta_{z}$ yields (9.3). For $z<0$ the proof is analogous.

Note that $\tilde{\kappa}$ is injective only on $\left(-\frac{\pi}{2}, \frac{\pi}{2}\right) \times(0, \pi)$. Still, $\tilde{\kappa}$ can be extended to $\tilde{D}:=\left(-\frac{\pi}{8}, \frac{5}{8} \pi\right) \times\left(\frac{\pi}{8}, \frac{7}{8} \pi\right)$. This means $\kappa_{1}$ can be extended to $\hat{\kappa}_{1}$ onto $\phi(\tilde{D})=$ $\left(\frac{1}{4}, 1+\frac{1}{4}\right)^{2}$. Hence, $\kappa_{1}$ satisfies Remark 8.2 with $\varepsilon_{i}^{a}=\varepsilon_{i}^{b}=\frac{1}{4}$ for $i=1,2$. The remaining patches $\Gamma_{i}$ and their parametrizations $\kappa_{i}:(0,1)^{2} \rightarrow \Gamma_{i}$ for $i=2, \ldots, 6$ are defined by rotating both the patches and the parametrizations by multiples of $\frac{\pi}{2}$. As we have a patch centered at each of the points $(1,0,0),(-1,0,0),(0,1,0)$, $(0,-1,0),(0,0,1),(0,0,-1)$, it is convenient to identify a patch with its center.

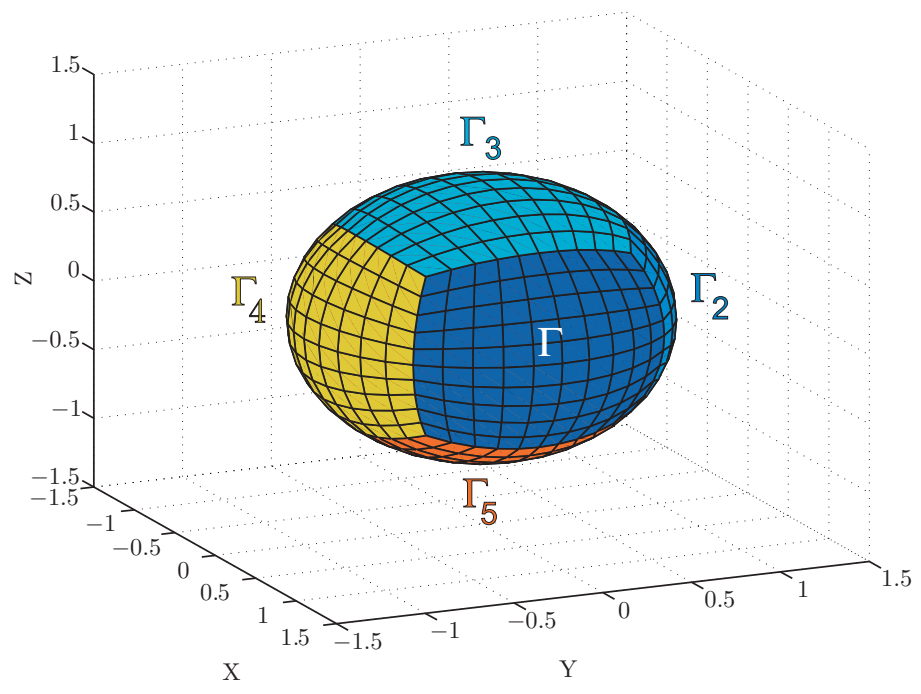

Figure 11. The sphere $S^{2}$ plotted by the current implementation with the visible Patches 1 through 5. Patch 6 is not visible. 
So $\Gamma_{x}$ will denote the patch centered at $x$ for the moment. We then see in Figure 11 that the numbering

$$
\begin{array}{ccrl}
\Gamma_{1} & =\Gamma_{(1,0,0)}, & \Gamma_{2} & :=\Gamma_{(0,1,0)}, \\
\Gamma_{3}:=\Gamma_{(0,0,1)}, & \Gamma_{4}:=\Gamma_{(0,-1,0)}, \\
\Gamma_{5}:=\Gamma_{(0,0,-1)}, & \Gamma_{6}:=\Gamma_{(-1,0,0)}
\end{array}
$$

follows the numbering rules presented in Subsection 8.1 The parametrizations of the $\kappa_{i}$ are then given by

$$
\begin{gathered}
\kappa_{2}=\left(\begin{array}{ccc}
0 & -1 & 0 \\
1 & 0 & 0 \\
0 & 0 & 1 \\
0 & 1 & 0 \\
-1 & 0 & 0 \\
0 & 0 & 1
\end{array}\right) \circ \kappa_{1}, \quad \kappa_{3}=\left(\begin{array}{ccc}
0 & 0 & -1 \\
0 & 1 & 0 \\
1 & 0 & 0 \\
0 & 0 & 1 \\
0 & 1 & 0 \\
-1 & 0 & 0
\end{array}\right) \circ \kappa_{1}, \quad \kappa_{5}=\kappa_{1}, \\
\kappa_{4}, \\
\kappa_{6}=\left(\begin{array}{ccc}
-1 & 0 & 0 \\
0 & -1 & 0 \\
0 & 0 & 1
\end{array}\right) \circ \kappa_{1} .
\end{gathered}
$$

The plot of the sphere can be seen in Figure 11.

9.2. Plotting the wavelets. We can now generate plots of the full biorthogonal wavelet bases of the Sobolev spaces $H^{m}\left(S^{2}\right)$ of the sphere for arbitrary $m$. As the results of the optimization of the Hestenes extension in Section 7 show that the Hestenes extension is very well conditioned for $m=1$, we will focus on this case.

There is a vast amount of basis functions already on the coarsest level. To get an idea of the number of essentially different types of wavelets, we quickly summarize the different types incorporated: Both the primal and the dual basis contain functions which are tensor products of unidirectional generators and wavelets. A number of those unidirectional generators and wavelets is adapted to the boundary, and left boundary functions are not necessarily symmetric to left boundary

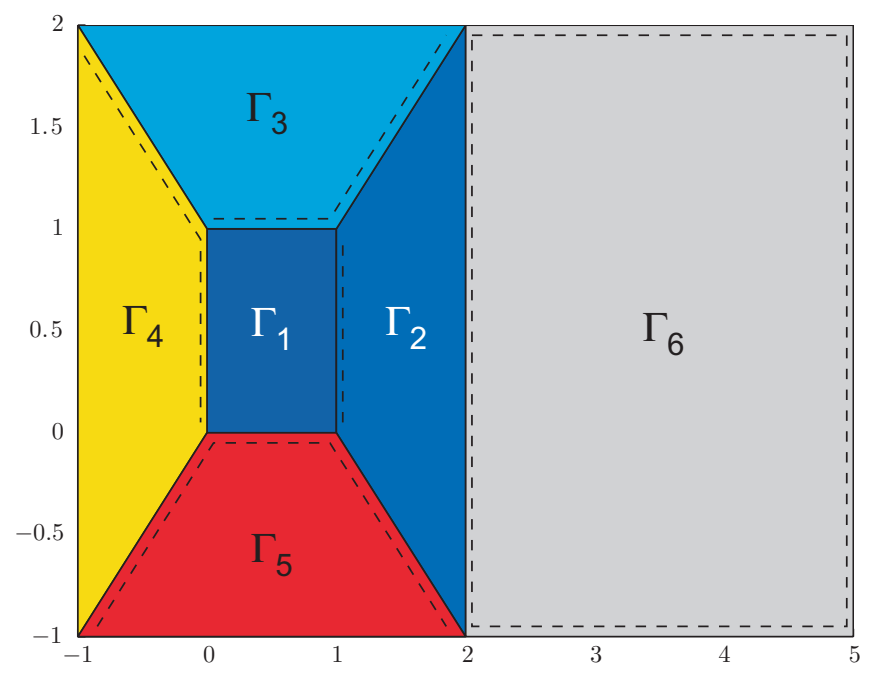

FiguRE 12. Flattened sphere with inflow boundary indicated by dashed lines. 
functions due to the varying boundary conditions. Finally there is one type of unidirectional generator and wavelet which does not intersect the boundary. This would amount to plotting at least $2 \times 4 \times 9 \times 6=432$ wavelets to get an idea of the wavelet bases - the factor 2 corresponds to the primal and dual functions, the factor 4 refers to the different choices of tensor products of generators and wavelets in each direction. The basis contains wavelets of type $\phi(x) \phi(y), \psi(x) \phi(y), \phi(x) \psi(y)$, and $\psi(x) \psi(y)$, where $\phi$ stands for a generator and $\psi$ for a wavelet. The factor 9 results from the location of the plotted wavelet in the patch. Due to the possibly asymmetric boundary conditions, there is a wavelet in each corner of a patch as well as a wavelet in the middle of each edge and one central wavelet, totaling in $4+4+1=9$ wavelets for each patch. Finally there are six patches, explaining the last factor. Plots of all these wavelets are provided in $[\mathbf{S}$.

Here we pick two wavelets which we plot in detail. The first wavelet $\psi$ is a primal wavelet living in the corner of $\Gamma_{1}$ that adjoins to $\Gamma_{2}$ and $\Gamma_{5}$, both lying in the outflow domain of $\Gamma_{1}$. So due to Remark 8.5, this wavelet has to be extended both to $\Gamma_{2}$ and $\Gamma_{5}$ (see Figure 12 for the neighbour relations). Figure 13 shows $\psi$ and the extension $E_{1} \psi$ to the outflow patches $\Gamma_{2}$ and $\Gamma_{5}$. Note that although $\Gamma_{3}$ and $\Gamma_{4}$ belong to the outflow of $\Gamma_{1}$ as well, the extension $E_{1} \psi$ is zero on those patches. Due to the support of the cutoff function incorporated in the Hestenes extension, only function values that are within a distance of $\varepsilon_{i}^{a}=\varepsilon_{i}^{b}=\frac{1}{4}$ (see Remark 8.2) of the outflow patch are regarded for the extension. The second wavelet $\tilde{\psi}$ is a dual wavelet living in the corner of $\Gamma_{3}$ that adjoins to $\Gamma_{2}$ and $\Gamma_{1}$, both lying in the inflow of $\Gamma_{3}$. As dual wavelets have free boundary conditions on the inflow (see Remark 8.4) $\tilde{\psi}$ has to be extended to these patches by $P_{3}^{*}$. Figure 14 shows $\tilde{\psi}$ and the extension to $\Gamma_{2}, \Gamma_{1}$.
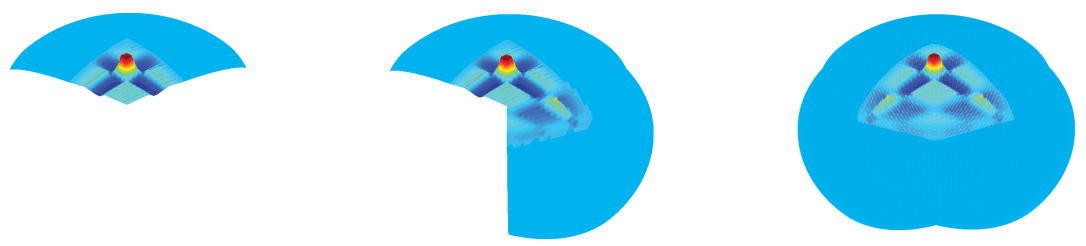

Figure 13. A primal wavelet on $\Gamma_{1}$ (left) being extended to the outflow patches $\Gamma_{2}$ (middle) and $\Gamma_{5}, \Gamma_{2}$ (right).
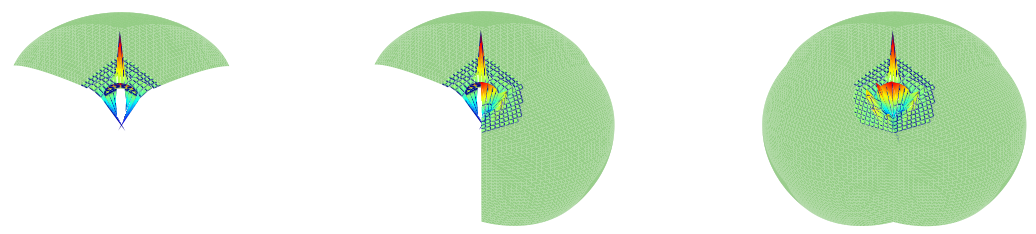

Figure 14. A dual wavelet on $\Gamma_{3}$ (above) being extended to the inflow patches $\Gamma_{2}$ (bottom left) and $\Gamma_{1}, \Gamma_{2}$ (bottom right). 


\section{ACKNOWLEDGMENTS}

We would like to thank Felix Otto for proposing the idea of the norm minimizing extension in Subsection 4.2. Marcel Arndt for suggesting the implicit gradient introduced in Remark 10.1 of [KS], and Helmut Harbrecht for supplying the wavelets with complementary boundary conditions on the interval. Also, Jan Sahner would like to thank Andreas Klein for his various helpful remarks. We thank an anonymous referee for helpful suggestions.

\section{REFERENCES}

[A] R. Adams, Sobolev Spaces, Academic Press, 1975. MR0450957 (56 \#9247)

[B] D. Bertsekas, Nonlinear Programming, Athena Scientific, 1999.

[Bo] C. de Boor, A Practical Guide to Splines, Applied Mathematical Sciences 27, Springer, 2003. MR0507062 (80a:65027)

[CF] Z. Ciesielski, T. Figiel, Spline bases in classical function spaces on compact $C^{\infty}$ manifolds: Part I and II, Studia Mathematica, 1983, 1-58 and 95-136. MR0728195 (85f:46060a) and MR0730015 (85f:46060b)

[CTU] C. Canuto, A. Tabacco, K. Urban, The wavelet element method, part I: Construction and analysis, Appl. Comput. Harm. Anal. 6, 1999, 1-52. MR1664902 (99k:42055)

[DKU] W. Dahmen, A. Kunoth, K. Urban, Biorthogonal spline wavelets on the intervalStability and moment conditions, Appl. Comput. Harmon. Anal. 6, No.2, 1999, 132-196. MR:1676771 (99m:42046)

[DS] W. Dahmen, R. Schneider, Wavelets on manifolds I: Construction and domain decomposition, SIAM J. Math. Anal. 31, No. 1, 1999, 184-230. MR.1742299(2000k:65242)

[DS1] W. Dahmen, R. Schneider, Composite wavelet bases for operator equations, Math. Comp. 68, 1999, 1533-1567. MR.1648379 (99m:65122)

[DS2] W. Dahmen, R. Schneider, Wavelets with complementary boundary conditions - function spaces on the cube, Results in Mathematics 34, 1998, 255-293. MR1652724 (99h:42057)

[DSt] W. Dahmen, R. Stevenson, Element-by-element construction of wavelets satisfying stability and moment conditions, SIAM J. Numer. Anal. 37, No. 1, 1999, 319-352. MR1742747 (2001c:65144)

[H] M. R. Hestenes, Extension of the range of a differentiable function, Duke Math. J. 8, 1941, 183-192. MR0003434(2,219c)

[Ha] H. Harbrecht, Wavelet Galerkin Schemes for the Boundary Element Method in Three Dimensions, Dissertation, Technische Universität Chemnitz, 2001.

[IGPM] A. Barinka, T. Bartsch, K. Urban, J. Vorloeper, The Multilevel Library, Institut für Geometrie und Praktische Mathematik, RWTH Aachen, 2001.

[KS] A. Kunoth, J. Sahner, Wavelets on manifolds: An optimized construction (extended version), SFB 611 Preprint \#163, Universität Bonn, July 2004, revised, April 2005, available at http://www.ins. uni-bonn.de/ kunoth/papers/papers.html

[S] J. Sahner, On the Optimized Construction of Wavelets on Manifolds, Diploma Thesis (in English), Universität Bonn, September 2003.

Institut für Numerische Simulation, Universität Bonn, Wegelerstr. 6, 53115 Bonn, GERMANY

E-mail address: kunoth@ins.uni-bonn.de

Konrad-Zuse-Zentrum für Informationstechnik Berlin (ZIB), TAKustr. 7, 14195 Berlin, Germany

E-mail address: sahner@zib.de 